\title{
A Less Invasive Approach of Medial Meniscectomy in Rat: A Model to Target Early or Less Severe Human Osteoarthritis
}

\author{
Subhash C Juneja ${ }^{1 *}$, Manuela Ventura², Gregory D Jay ${ }^{3,4}$ and Christian Veillette ${ }^{1}$
}

${ }^{1}$ Arthritis Program, Orthopaedic Surgery, Toronto Western Hospital, 399 Bathurst St, Toronto, ON, Canada ${ }^{2}$ STTARR Innovation Centre, Princess Margaret Cancer Centre, 101, College St, Toronto, ON, Canada

${ }^{3}$ Department of Emergency Medicine, Rhode Island Hospital, Providence, RI, 593 Eddy St, Providence, RI 02903, USA

${ }^{4}$ School of Engineering, Brown University, Providence, RI, 02912 USA

\begin{abstract}
Objectives: The existing medial meniscectomy (MMx) procedure in rodents involves transection of $\mathrm{MCL}$ and wide opening of the knee capsule followed by meniscus transection that results into articular cartilage degeneration and causes significant changes in subchondral bone at specific post-MMx period. These animals serve as experimental osteoarthritis (OA) models.

Structurally, knee is very complex. There may be a need to uncouple articular cartilage degenerative changes from subchondral bone degenerative changes in experimental OA models. Therapeutical drugs/agents, designed to treat articular cartilage degenerative changes of $O A$ knee, may not be effective to treat subchondral bone degenerative changes or vice versa.

The purpose of the study was to modify the existing MMx procedure to a less invasive procedure so that it causes only articular cartilage degenerative OA changes and no subchondral bone changes. This will serve as an early OA model to target degenerative changes in articular cartilage in human.

Methods: Ten 8-9 weeks old male athymic nude rats underwent less invasive MMx on right knee; the left knee served as unoperated control. The surgery involved no transection of MCL and opening of knee capsule wide. The medial meniscus was pulled out from a slit made on the medial aspect of the knee capsule, and was transected. At 10 weeks post-MMx, animals were sacrificed and knees were assessed.

Results: There was no significant alteration in bone strength parameters (BV/TV, Tb.Th, Tb.Sp, Tb.N, Tb.Pf, cortical wall thickness) in subchondral bone in the less invasive MMx knee as analyzed by $\mu \mathrm{CT}$. X-ray radiography did not indicate the presence of any osteophyte at the knee margins. On the other hand, the less invasive MMx caused degenerative changes in articular cartilage as follows: fibrillation and thinning in the medial tibia; narrowing of medial knee joint; reduced proteoglycans shown by safranin-O staining; decreased metachromasia by PSR staining; alteration in collagen fibril thickness under cross-polarization light. Immunohistochemical expression was reduced (COL-II, aggrecan), increased (NITEGE, Col2-3/4m, COL-X, and MMP13) or imbalanced (lubricin) in articular cartilage thickness of less invasive MMx knee.

Conclusion: The less invasive MMx procedure, in rat, can be a model for early or less severe human OA due to articular cartilage degenerative changes only and with no subchondral bone degenerative changes in the knee synovial joint. The therapeutical drugs/agents designed to repair articular cartilage may be suitable for this model and may lead to treatment of early or less severe human OA.
\end{abstract}

Keywords: Osteoarthritis; Medial meniscectomy; Rats; $\mu \mathrm{CT}$; Immunohistochemistry; Articular cartilage; Subchondral bone

\section{Introduction}

Osteoarthritis $(\mathrm{OA})$ is one of the leading causes of disability in the elderly. OA is defined by the American College of Rheumatology as a "heterogeneous group of conditions that lead to joint symptoms and signs which are associated with defective integrity of articular cartilage, in addition to related changes in the underlying bone at the joint margins". The OA progresses with age, 30 to $50 \%$ of adults over the age of 65 years suffer from OA. Radiography of multiple joints (knee, hip, spine, hand) shows OA in at least one joint in over $80 \%$ of older adults. However, only about half of people with radiographic OA experience significant symptoms. And also not all the older adults with joint pain have radiographic evidence of OA in the affected joint [1]. Diagnosis is usually confirmed by using X-ray with joint space narrowing, osteophytes formation and subchondral sclerosis [2]. Osteophytes generally appear at joint margins, initially as outgrowths of cartilage and those subsequently undergo endochondral ossification supported by growth factors including TGF $\beta$ [3].
Medial meniscus is attached to the tibial plateau via anterior and posterior horn by insertions that hold the meniscus in place during weight bearing [4]. The menisci of the knee are important load distributors and shock absorbers in the joint. Meniscectomy causes instability to the knee joint with an increased risk of OA [5]. Partial meniscectomy has been reported to increase the risk for the onset of OA by increasing stress and strain in the articular cartilage [6]. The

*Corresponding author: Subhash C Juneja, BSc, MSc, PhD., Arthritis Program, Orthopedic Surgery, Toronto Western Hospital, 399 Bathurst St, Toronto, ON M5T 2S8 Canada, Tel: (416) 422-0438; Fax:(416-603-3437; E-mail: sjuneja.phd@gmail.com

Received February 01, 2016; Accepted February 16, 2016; Published February 27,2016

Citation: Juneja SC, Ventura M, Jay GD, Veillette C (2016) A Less Invasive Approach of Medial Meniscectomy in Rat: A Model to Target Early or Less Severe Human Osteoarthritis. J Arthritis 5: 193. doi:10.4172/2167-7921.1000193

Copyright: @ 2016 Juneja SC, et al. This is an open-access article distributed under the terms of the Creative Commons Attribution License, which permits unrestricted use, distribution, and reproduction in any medium, provided the original author and source are credited. 
external medial tibial cartilage is vulnerable to thinning once the meniscus extrudes and its surface is in direct, non-physiological, cartilage-cartilage contact with femur [7]. Papalia et al. summarized that meniscectomy is a risk factor of OA though the extent of cartilage degeneration depends upon the 'minimal invasion to open total meniscectomy', gender, and other factors [8]. There is no disease modifying OA drug on the market that slows down OA progression or induces neo-formation of the articular cartilage. In search of therapeutical solutions, surgical joint instability models are of great interests. Several animal models of OA, spontaneously mutated, genetically modified or created by multiple manipulations, have been described in different species. Among them are: dog partial MMx [9]; dog anterior cruciate ligament transection (ACLT; [10]); rat medial meniscus tear [11]; rat ACLT [12]; gene mutation model in mice, e.g., mutation in COL-II [13]; spontaneous development of OA in male STR/ort mice [14]; surgically or chemically-induced OA in mice, e.g., DMM (destabilization of knee by MMx) model [15], or collagenase injection (CIA) in knee capsule [16]; spontaneous OA in Hartley albino guinea pigs [17]; meniscus tear-induction in guinea pig [18]; meniscustear in rabbit [19]; and spontaneous OA in non-human primates [20].

In rat and mouse, MMx surgery is usually conducted first by transecting the medial collateral ligament (MCL) fully or partially followed by wide opening of the knee capsule, and finally excising the medial meniscus $[15,21,22]$. Following the MMx procedure, a substantial subchondral bone changes occurred in rat knee at 8-weeks post-MMx as determined by $\mu \mathrm{CT}$ along with articular cartilage degeneration [23]. For analyzing subchondral bone damages, McErlain and team used MRI and $\mu \mathrm{CT}$ and showed that $75 \%$ of 4 -weeks postACLT rats had at least 1 subchondral bone cyst [24]. The uncoupling of cartilage damage and subchondral trabecular bone changes in OA is suggested [25], a separate set of markers need to be considered in experimental OA. A model that causes articular damage and less or no subchondral bone damage can serve better choice for experimental OA for cartilage repair.

The purpose of the current investigation was to modify the existing MMx procedure into less invasive procedure that causes predominantly articular cartilage degeneration and not the subchondral bone changes in rat. The procedure was conducted in which MCL of the knee joint was not transected, knee capsule was not opened wide, and the MMx was accomplished successfully with degenerative changes in the articular cartilage of medial knee joint but with non-significant changes in the subchondral bone strength parameters. By using this modified procedure, we assumingly avoided most of the additional synovial inflammation as compared to wide-opened knee capsule surgery. Synovium inflammation has been directly linked to enhanced OA [26]. Macrophages from the synovial lining have been shown to cause inflammation and cartilage damage in experimental arthritis model [27]. The less invasive MMx in rat may reflect post-traumatic OA model and not post-inflammatory OA model serve as an early OA model to target degenerative changes in articular cartilage in human. The purpose of the study is to use less invasive MMx procedure in rat in search of an experimental model for early or less severe human OA. We used athymic nude rats instead of normal rats for this study for the reason that this rat is becoming an experimental animal of choice for human cells transplantation studies to avoid rejection, and will also serve as human stem cells transplantations in our future experiments.

\section{Methods}

\section{Medial meniscectomy (MMx)}

Animals care: Male homozygous athymic nude rats, also called
$\mathrm{NIH}$ nude rats (Crl:NIH-Foxn1rnu; rnu/rnu), were purchased from Charles Rivers, USA (1.800.LABRATS). Animal use protocol (AUP) was approved by animal resources center (ARC) of University Health Network, Toronto. Upon arrival, rats were allowed to acclimatize for three days to new housing. Animals were provided with sterile housing, food and drinking water with restricted entry.

Surgeries: Surgeries were conducted on rats at the age of 8-9 weeks using eye loupes (4.9x, Surgical Acuity). Rats were anesthetized using $5 \%$ isoflurane in oxygen (inhalation anesthetic) and maintained at $2 \%$ isoflurane during surgery. Pre- and post-operative care was provided to animals. Tear gel (Alcon Tear-Gel Liquid Eye Gel) was applied to rat's eyes immediately after anesthetization prior to surgery. Rats received one injection of analgesia (buprenorphine, $0.03 \mathrm{mg} / \mathrm{kg}$ ) just before the beginning of surgery and two injections daily (one in morning and one in the evening) for three days post-surgery. Animals were kept hydrated by injecting sc saline $(\approx 2-3 \mathrm{ml})$ at the back area before beginning of the surgery. Surgeries were conducted on a clean bench covered with sterile drape sheets cushioned and warmed underneath with a pad equipped with T/Pump Warm Water Recirculator (STRYKER). Knees and surrounding area was shaved, cleaned with iodine surgical scrub (7.5\% iodine), $70 \%$ isopropanol and $10 \%$ Providone iodine (equivalent to $1 \%$ iodine) in sequence. MMx was conducted on the right knee $(\mathrm{N}=10)$ of the rat with less invasive approach as compared to the methods used by earlier researchers in rat or mouse [15,21,22]. Left knees served as unoperated controls $(\mathrm{N}=10)$. A longitudinal incision was made on the anterior aspect of the right knee with a blade no. 15 (M90-15, Almedic)/scalpel handle no. 3. The skin, underneath and around the knee, was gently dislodged from the subcutaneous layer to make a small pocket flap by using few backward strokes of scissors. A medial peripatellar incision was made to expose MCL along with the outer surface of knee capsule. The later was cleaned with cotton gauze to locate the position of medial meniscus that was visible through capsule's translucent wall under high-powered eye loupes (Figure 1A). The meniscus was gently dislodged from medial femur and medial tibia with the help of a micro knife (10316-14, Micro knife $22.5^{\circ}$, Fine Scientific Tools; Figure 1A). The meniscus was pulled out with a pair of fine-toothed forceps (Figure 1B), cut first at one end and than pulled out, and cut at the second end (Figures 1C1, 1C2 and 1D). The slit in the medial knee capsule was washed with $0.5 \mathrm{ml}$ PBS (D8662, Dulbecco's phosphate-buffered saline, Sigma-Aldrich) and closed with 2 interrupted stitches using 6-0 PolysorbTM suture (Polysorb, UL-201, Covidien). The subcutaneous layer was sutured using 5-0 PolysorbTM (UL-202, Covidien) using three interrupted stitches. Skin was closed by uninterrupted intradermal suturing system using Monocryl suture (Y303, Ethicon). Finally the sutured skin was glued with a thin layer of tissue adhesive (Vetbond, 3M). Animals were placed under warm lamp until they moved freely in the cage $(\approx 5-10 \mathrm{~min})$. Animals were monitored twice daily for first 3 days; daily for next 4 days, and later twice a week till the end of animal experiment.

\section{$\mathrm{X}$-ray radiography and $\mu \mathrm{CT}$ imaging of rat knee joints}

At 10 -week post-MMx, rats were euthanized using $\mathrm{CO}_{2}$ gas anesthesia. Whole body and separated individual hind limbs were radiographed using VXvue digital imaging system following the instruction manual. Specimens were placed on the detector plate at a distance of 30 inches from the X-ray emitter. X-ray dose for the generator was set at $\mathrm{kV}(\mathrm{kVP}=70)$ and $\mathrm{mAs}(1.000)$. X-ray machine was handled and operated in a designated area approved by Radiation Hazards and Protection division, Ministry of Labor, Ontario, Canada. The digital images were downloaded. The representative images are 


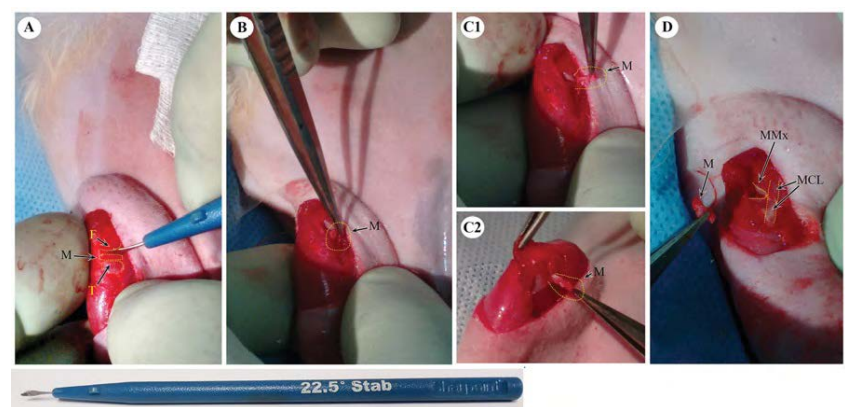

Figure 1: Less invasive surgical procedure. MMx of right knee of rat was conducted in four steps: Locating the medial meniscus on the surface of knee capsule without opening the knee capsule through translucent surface; and dislodging the meniscus from femur anteriorly and tibia posteriorly with a micro knife (A); Pulling of medial meniscus from the knee capsule outward with a pair of fine toothed forceps through a small slit (B); Cutting the anterior end of medial meniscus with the micro knife and pulling it outward $(C 1, C 2)$; Cutting of second end of medial meniscus from knee capsule and leaving behind the MMx knee (D). F, T, M, MCL indicates femur, tibia, meniscus, medial collateral ligament, respectively. Towards the bottom of the figure, a micro knife is shown.

shown in Figure 2. Knee joints were cut separated with a dremel, fixed in 10\% neutral buffered formalin (HT-5011, Sigma-Aldrich) with two changes during $72 \mathrm{~h}$, washed in PBS (2x, $10 \mathrm{~min})$, transferred to $70 \%$ ethanol and processed for $\mu \mathrm{CT}$ imaging. The knee joints were scanned using a small-animal Inveon CT scanner (Siemens Preclinical Solutions, Knoxville, TN), with the parameters shown in Table 1 . The reconstructed datasets were analyzed with the Inveon Research Workplace software (v4.0). Histomorphometric analysis of subchondral bone (medial tibia and femur) was performed by 3 -D volume of interest (VOI). The VOI included the subchondral trabecular bone starting below the subchondral plate extending $(0.5$ $\mathrm{mm}$ in depth) towards the growth plate, while excluding both the cortical bone and growth plate interface. For analysis of medial tibia and femur cortical bone, VOI regions included the load-bearing region of the medial cortical plates. ROIs were designed throughout the full width and thickness of the cortical layer, and measured $2.5 \mathrm{~mm}$ in length from the anterior-posterior side (Figure 3 and Table 1). Scanned images of MMx and control knee are shown in Figure 4. The following bone strength parameters were calculated: BV/TV, Tb.Th, Tb.Sp, Tb.N, Tb.Pf, cortical wall thickness (Figure 5).

\section{Histological analysis}

After $\mu \mathrm{CT}$ imaging, knee joints were rinsed in PBS, decalcified in a very mild formic acid-based decalcifier (15x vols; 1414-1, Immunocal ${ }^{\mathrm{TM}}$, StatLab Medical Products) for 10 days with 4 changes, equilibrated with PBS for 2 hours, and embedded in paraffin blocks. Coronal sections (4 $\mu \mathrm{m}$ thickness) of knee joints were cut. For IHC, slides with paraffin tissue sections were heated at $55-58^{\circ} \mathrm{C}$ for 4 hours to avoid falling during multiple steps of the staining procedure. Paraffin sections, for all the histological and IHC stainings, were deparaffinized, cleared and hydrated by passing the slides thorough Histo-Clear $\mathrm{II}^{\circledR}(3 \mathrm{x}, 3$ min, 64111-01, EM Sciences), and decreasing concentration of ethanol [ $\left(100 \%, 95 \%, 70 \%, 0 \%\left(\mathrm{dH}_{2} \mathrm{O}\right), 3 \mathrm{x}, 3 \mathrm{~min}\right.$ each $]$, and finally immersed in fresh $\mathrm{dH} 2 \mathrm{O}$. Deparaffinized hydrated knee sections were used in the following procedures.

H\&E staining: Coronal sections of rat knee were stained using $\mathrm{H} \& \mathrm{E}$ protocol. For that, the deparaffinized hydrated sections were stained with Mayer's hematoxylin solution (MHS-16, Sigma-Aldrich) for $10 \mathrm{~min}$, washed in warm tap water for $10 \mathrm{~min}$, rinsed once in distilled water, dipped in $95 \%$ ethanol $(10 \mathrm{x}, 1 \mathrm{sec})$, and stained with eosin (HT110316, Eosin Y solution with phloxine, Sigmaaldrich) for 45 secs. The slides were dehydrated in 95\% ethanol (3x, $3 \mathrm{~min}$ ), 100\% ethanol ( $3 \mathrm{x}, 3 \mathrm{~min})$, cleared in Histo-Clear $\mathrm{II}^{\circ}(3 \mathrm{x}, 3 \mathrm{~min})$ and mounted in Omnimount ${ }^{\mathrm{TM}}$ mounting medium (17997, EM sciences). Mounted slides were scanned at 20x magnification using Aperio ScanScope XT. The images were opened in a PC computer using the image analysis software Aperio ImageScope (v10). The images were saved as tif files. Nuclei presented blue and cytoplasm as pink coloration.

Safranin O staining: Safranin O binds to glycosaminoglycan and the staining represents the presence of proteoglycans in the cartilage. The deparaffinized hydrated knee sections were stained in freshly reconstituted Weigert's iron hematoxylin ( $1 \mathrm{x}, 5 \mathrm{~min})$, rinsed in changes of $\mathrm{dH}_{2} \mathrm{O}$ till leaching of blue coloration stopped. Sections were differentiated in $1 \%$ acid-alcohol $\left(2\right.$ secs), rinsed in $\mathrm{dH}_{2} \mathrm{O}(3 \mathrm{x})$, treated with $0.02 \%$ Fast Green ( $1 \mathrm{x}, 15 \mathrm{~min})$, cleared in $1 \%$ acetic acid (10 secs), and finally treated with $1 \%$ safranin O (20800, EM Sciences) for 10 min. The sections were dehydrated in $95 \%$ ethanol ( $4 \mathrm{x}, 15$ secs) and $100 \%$ ethanol $(4 \mathrm{x}, 15 \mathrm{secs})$ and cleared in Histo-Clear $\mathrm{II}^{\mathbb{B}}(3 \mathrm{x}, 3 \mathrm{~min})$ and mounted in Omnimount ${ }^{\mathrm{TM}}$ mounting medium (17997, EM sciences). Proteoglycans stains orangered, cytoplasm as gray green and nuclei as black. Mounted slides were scanned, and saved as tif files as described earlier.

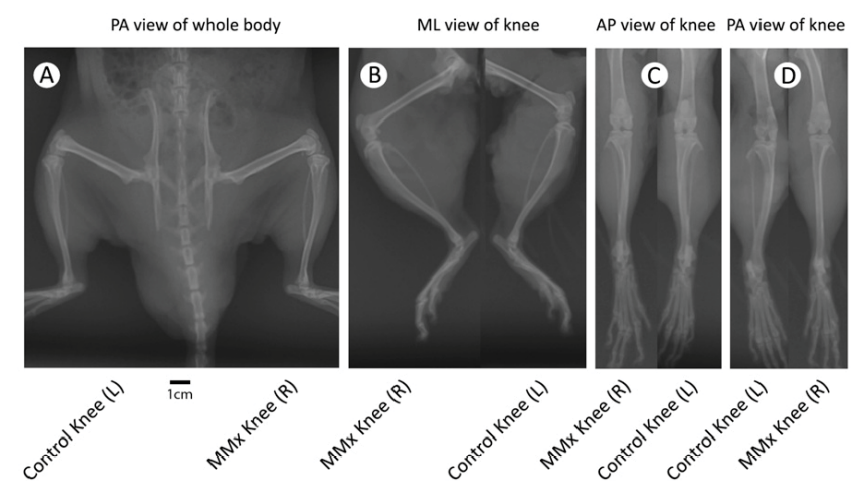

Figure 2: $X$-Ray radiography of rat knee. Posterior-anterior (PA) view of whole body (A); ML (medial-lateral) view of knee (B); AP (anterior-posterior) view of knee (C); PA (posterioranterior) view of knee (D). $L=$ left, $R=$ right.

\begin{tabular}{|c|c|}
\hline \multicolumn{2}{|c|}{ CT scan parameters } \\
\hline Total Rotations & $360^{\circ}$ \\
\hline Rotation Steps & 180 \\
\hline Number of Calibrations & 20 \\
\hline Settle Time & $200 \mathrm{~ms}$ \\
\hline FOV & $256 \times 3072$ \\
\hline Binning & 2 \\
\hline Exposure Time & $1500 \mathrm{~s}$ \\
\hline Average Frames & 1 \\
\hline Effective Pixels size & $18 \mu \mathrm{m} .40$ \\
\hline System magnification & High \\
\hline Voltage & $80 \mathrm{KV}$ \\
\hline Current & $500 \mu \mathrm{A}$ \\
\hline & Thresholds \\
\hline$-368.664-350.793$ & Trabecular \\
\hline -839.079 -350.793 & Bone marrow \\
\hline
\end{tabular}

Table 1: $\mu$ CT scan parameters used in the study. 
Citation: Juneja SC, Ventura M, Jay GD, Veillette C (2016) A Less Invasive Approach of Medial Meniscectomy in Rat: A Model to Target Early or Less Severe Human Osteoarthritis. J Arthritis 5: 193. doi:10.4172/2167-7921.1000193

Page 4 of 17

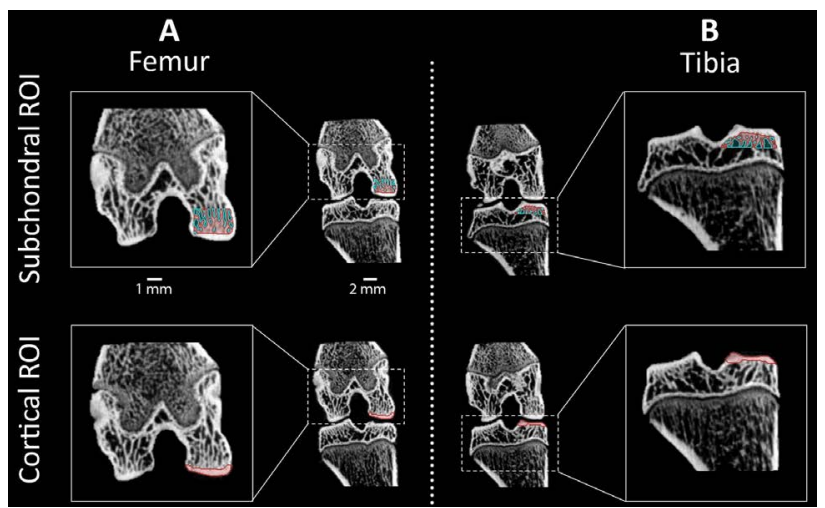

Figure 3: $\mu C T$ scan of rat knee. Femur $(A)$ and tibia $(B)$ shows subchondral and cortical ROI. VOI regions include the load-bearing region of the medial cortical plates. ROIs were designed throughout the full width and thickness of the cortical layer, and measured $2.5 \mathrm{~mm}$ in length from the anteriorposterior side. VOI histo-morphometric analysis of subchondral bone (medial tibia and femur): The VOI included the subchondral trabecular bone starting below the subchondral plate, and extending distally $(0.5 \mathrm{~mm}$ in depth) towards the growth plate, excluding both the cortical bone and growth plate interface.

Picrosirius red (PSR) staining, and Polarization light microscopy: The deparaffinized hydrated knee sections were stained with PSR stain kit following the manufacturer's instructions (24901,
Polysciences, Inc.). PSR-stained sections were dehydrated by passing through increasing concentration of ethanol $(70 \%, 95 \%, 100 \%, 3 \mathrm{x}, 3$ min each) and cleared through Histo-Clear $\mathrm{II}^{\oplus}(3 \mathrm{x}, 3 \mathrm{~min})$. The sections were mounted in Omnimount ${ }^{\mathrm{Tw}}$ mounting medium. Mounted slides

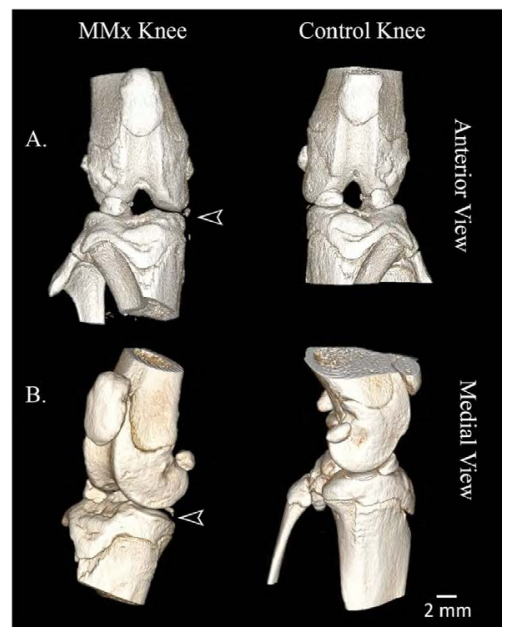

Figure 4: $\mu \mathrm{CT}$ scan. Anterior view (A) and medial view (B) of MMx knee (images on left) and control knee (images on right); indication of a space between medial femur and medial tibia (an arrow head) in MMx knee.

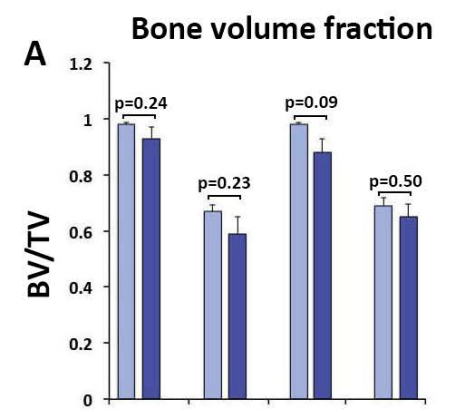

Trabecular pattern factor

D
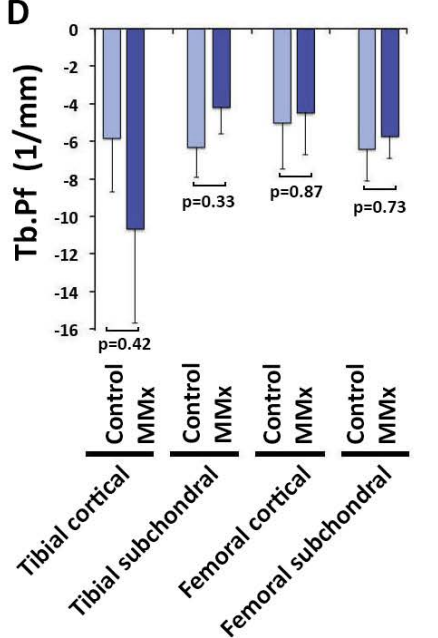

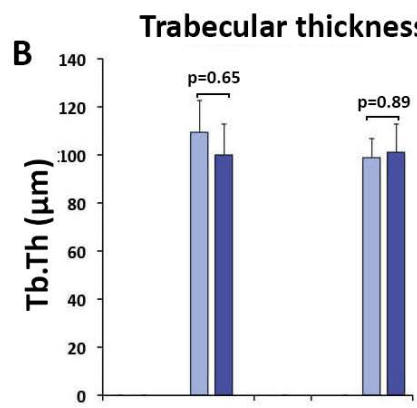

Trabecular number

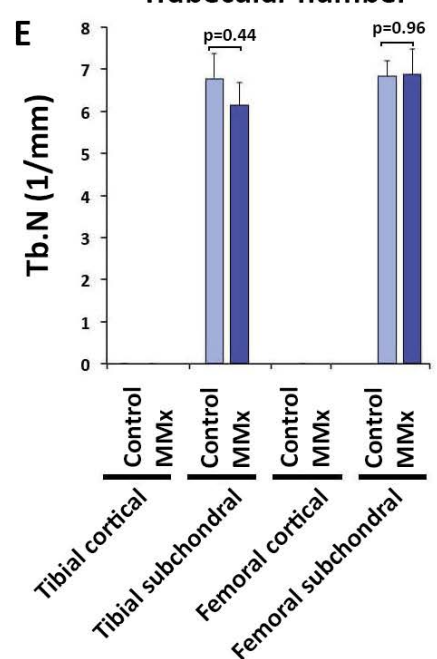

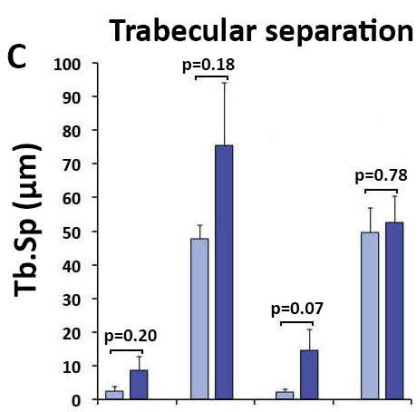

\section{Cortical wall thickness}

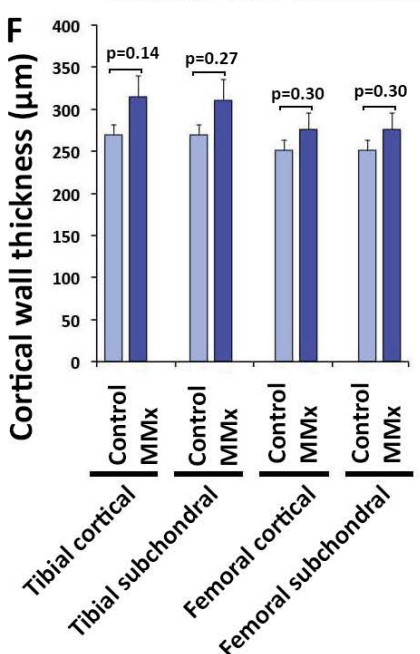

Figure 5: $\mu \mathrm{CT}$ scan analysis. Effect of less invasive MMx on tibial cortical and subchondral, femoral cortical and subchondral ROls in 8-9 wks old rat knee at 10 wks post-surgery. After 3-D reconstruction, BV/TV(A), Tb.Th (B), Tb.Sp (C), Tb.Pf (D), Tb.N (E) and cortical wall thickness (F) parameters were acquired and analyzed. $\mathrm{MMx}$ at right knee; Control as unoperated left knee. 
were viewed under upright brightfield and polarization Olympus BX51 microscope. The collagen stains bright red color under light microscope, whereas, under polarization light, collagen shows different colors based on thickness of fibers: thick (red/orange/yellow), thin (green), and thick/thin mix (lime green-blue).

Immunohistochemistry (IHC): The deparaffinized and hydrated paraffin sections of knee joints were processed for antigen retrieval by enzymatic treatment as described in Table 2 . The sections were washed in $\mathrm{dH}_{2} \mathrm{O}(3 \mathrm{x}, 3 \mathrm{~min})$, treated with freshly diluted $3 \% \mathrm{H}_{2} \mathrm{O}_{2}(\mathrm{H} 1009$, Hydrogen peroxide solution, Sigma- Aldrich), and rinsed in $\mathrm{dH}_{2} \mathrm{O}$ (4x). The sections were incubated with blocking buffer (Table 2) for 30 min, replaced with primary antibody (Table 2) and incubated at $4^{\circ} \mathrm{C}$ for overnight. The sections were washed with PBS-T (Table 2; $4 \mathrm{x}, 5$ $\mathrm{min}$ ), treated with blocking buffer for $30 \mathrm{~min}$, replaced with secondary antibody (Table 2) and incubated for $30 \mathrm{~min}$ at room temperature. The sections were washed with PBS-T (4x, 5 min) and incubated with ABC reagent $[A B C$ reagent prepared $30 \mathrm{~min}$ before incubation (PK-6100, Elite ABC-HRP kit, Vector)] for $30 \mathrm{~min}$. The sections were washed with PBS- $\mathrm{T}(2 \mathrm{x}, 1 \mathrm{~min})$ and then with $\mathrm{dH}_{2} \mathrm{O}(4 \mathrm{x}, 1 \mathrm{~min})$. The light to dark brown color (i.e., peroxidase reaction) was developed using $0.4 \mathrm{ml}$ freshly diluted peroxidase substrate, diaminobenzidine (DAB; Sk-4105, ImmPACT ${ }^{\mathrm{TM}}$ DAB Substrate, Vector) on sections for 2-3 min while watching under the stereomicroscope depending upon the peroxidase activity. Immersing the slides in $\mathrm{dH}_{2} \mathrm{O}$ stopped the reaction. The slides, once rinsed in $\mathrm{dH}_{2} \mathrm{O}$, were stained in Meyer's hematoxylin (MHS16, Sigma) for $4 \mathrm{~min}$, rinsed in changes of $\mathrm{dH}_{2} \mathrm{O}$ till leaching of blue coloration stopped. The sections were dehydrated in the increasing concentrations of ethanol (70\%, 95\% and 100\% (3x, $3 \mathrm{~min}$, each), cleared in Histo-Clear $\mathrm{II}^{\circ}(3 \mathrm{x}, 3 \mathrm{~min})$ and mounted in Omnimount ${ }^{\mathrm{m}}$ mounting medium. Mounted slides were scanned and saved as tif files as described earlier. Human OA knee cartilage or human normal tracheal cartilage or post-natal day 1 (P1) mouse spine, were used as positive control during various histological or IHC procedures. The human OA knee specimens were obtained from two OA patients (age, 58, 64 year) undergoing knee joint replacement surgery at Toronto Western Hospital, Toronto. The protocol, to collect human specimens from patients, was approved by research ethics board (REB) committee of University Health Network, Toronto. Mouse tissue was obtained from a recently died P1 pup obtained from Animal Resources Center of University Health Network, Toronto. Paraffin sections of normal human (age, 58 year) tracheal cartilage were purchased (CAR01, Pantomics, Inc. Richmond, CA).

\section{Statistical analysis}

The $\mu \mathrm{CT}$ scan data was analyzed by one-way ANOVA using Tukey HSD all pair comparison (alpha=0.05). $\mathrm{N}=10$.

\section{Results}

\section{Medial meniscectomy (MMx)}

The medial meniscectomy was achieved successfully in rat by using a less invasive approach. The procedure did not require transection of MCL and opening of the knee capsule wide during the surgery, rather a small slit was made on the medial side of knee capsule to pull out the majority of medial meniscus (Figure 1). Rats recovered within one week post-surgery without complication, and remained healthy throughout the experimental period of 10 weeks post-surgery.

\section{$\mathrm{X}$-ray radiography, and $\mu \mathrm{CT}$ scan analysis of knee joints}

Images of X-ray radiographs showed that MMx was successful in rat using the less invasive procedure. There was no noticeable bone sclerosis or subchondral bone cyst in MMx knee (Figures 2A2D). There was a gap visible between medial femur and medial tibial plateau in MMx knee as compared to control counterpart indicating the absence of medial meniscus (Figures 2C and 2D). There was no noticeable osteophyte in MMx knee joint compartment. Cortical and subchondral ROIs of medial femur and medial tibia were chosen for $\mu \mathrm{CT}$ analysis and are shown as 3-D representation images (Figures 3A and $3 \mathrm{~B}$ ). The $\mu \mathrm{CT}$ scan of MMx knee showed a gap between medial femoral condyle and medial tibia confirming the absence of medial meniscus and success of the less invasive procedure (Figures $4 \mathrm{~A}$ and $4 \mathrm{~B})$. The analysis of the subchondral bone strength parameters is shown in Figures 5A-5F. BV/TV ratio, in MMx knee, decreased at all the 4 ROIs (tibial cortical, tibial subchondral, femoral cortical, femoral subchondral) as compared to control knee, though the change was not statistically significant (Figure 5A). Of all, the most affected ROI was femoral subchondral (p, 0.09). Tb.Th, measured at 2 ROIs (tibial

\begin{tabular}{|c|c|c|c|}
\hline Antigen & Primary antibody & Secondary antibody & Antigen retrieval \\
\hline Type II collagen (COL-II) & Mouse monoclonal, anti-COL II, II-II6B3, DSHB & $\begin{array}{l}\text { Biotinylated horse anti- mouse } \lg G \text {, rat } \\
\text { adsorbed, BA-2001, Vector }\end{array}$ & * \\
\hline $\begin{array}{l}\text { Denatured COL II } 3 / 4 \text { fragment } \\
(\text { Col } 2-3 / 4 \mathrm{~m})\end{array}$ & $\begin{array}{l}\text { Mouse monoclonal, Col2-3/4m antibody, 50-1011, IBEX } \\
\text { Pharmaceuticals Inc. }\end{array}$ & Same as above & ** \\
\hline Type- $X$ collagen (COL-X) & Mouse monoclonal, anti-COL X 2031501001, Quartett, GE & Same as above & * \\
\hline Lubricin (PRG4) & Mouse monoclonal, Anti- lubricin (9G3). & Gregory Jay's lab & * \\
\hline NITEGE & $\begin{array}{l}\text { Rabbit polyclonal antibody to aggrecan N-terminal neoepitope NITEGE } \\
\qquad(\text { BC13), NB100-74350, NOVUS }\end{array}$ & $\begin{array}{l}\text { Biotinylated horse anti- rabbit lgG, } \\
\text { BA-1100, Vector }\end{array}$ & $* * *$ \\
\hline Aggrecan & Rabbit polyclonal, aggrecan antibody, Ab36861, abcam & Same as above & *** \\
\hline MMP13 & $\begin{array}{l}\text { Rabbit Polyclonal, MMP13 } \\
\text { antibody, ab39012, abcam }\end{array}$ & Same as above & **** \\
\hline
\end{tabular}

Negative controls contained no primary antibody and contained normal lgG isotype control;

Each antibody was diluted in blocking buffer that constituted of PBS-T with $2 \%$ BSA (ALB-001, Albumin bovine serum fraction V, Bioshop) and $2 \%$ horse serum (16050122, GIBCO) or Donkey serum (D9663, Sigma-Aldrich); PBS-T was composed of D-PBS containing 0.05\% Tween-20 (TWN510, Bioshop). The antigen retrieval for knee sections and controls used were as follows:

${ }^{*}$ The hydrated tissue sections were treated with pepsin ( $\mathrm{P}-7000$, Sigma-Aldrich; $4 \mathrm{mg} / \mathrm{ml}$ in $\left.0.01 \mathrm{~N} \mathrm{HCl}\right)$ for 10 min at $37^{\circ} \mathrm{C}$, washed with $\mathrm{dH}{ }_{2} \mathrm{O}(4 \mathrm{x}, 1 \mathrm{~min})$, treated with hyaluronidase $\left(\mathrm{H}-3506\right.$, Sigma-Aldrich) solution at $1 \mathrm{mg} / \mathrm{ml}$ (in $0.1 \mathrm{M}$ phosphate buffer, $\mathrm{pH} 5.0$ ) for $30 \mathrm{~min}$ at $37^{\circ} \mathrm{C}$, and rinsed in PBS-T ( $3 \mathrm{x}, 2 \mathrm{~min}$ );

**The hydrated tissue sections were treated with hyaluronidase at $10 \mathrm{mg} / \mathrm{ml}$ (in $0.1 \mathrm{M}$ phosphate buffer, $\mathrm{pH} 5.0$ ) for $30 \mathrm{~min}$ at $37^{\circ} \mathrm{C}$, and rinsed in PBS-T ( $3 \mathrm{x}, 2 \mathrm{~min}$ );

${ }^{* * *}$ The hydrated tissue sections were treated with hyaluronidase at $25 \mathrm{mg} / \mathrm{ml}$ (in $0.1 \mathrm{M}$ phosphate buffer, pH 5.0 ) for $35 \mathrm{~min}$ at $37^{\circ} \mathrm{C}$, rinsed in PBS-T ( $4 \mathrm{x}, 1 \mathrm{~min}$ ), treated with chondroitinase ABC (C3667, Sigma-Aldrich; $0.125 \mathrm{Unit} / \mathrm{ml}$ in $0.01 \mathrm{M}$ citrate buffer, $\mathrm{pH} 6.0$ ) for $45 \mathrm{~min}$ at $37^{\circ} \mathrm{C}$, and rinsed in PBS-T (3x, $2 \mathrm{~min}$ );

${ }_{* * * *}$ The hydrated tissue sections were treated with Proteinase K [(25530-049, Invitrogen; $20 \mu \mathrm{g} / \mathrm{ml}$ in TE buffer (50mM TRIS with $1 \mathrm{mM}$ EDTA and 0.5\% Triton X-100, pH 8.0)] for $15 \mathrm{~min}$ at $37^{\circ}$ followed by slow cooling for $5 \mathrm{~min}$, and rinsed in PBS-T (3x, 2min). 
subchondral, femoral subchondral), did not differ between MMx and control knee (Figure 5B). Tb.Sp was higher at all the 4 ROIs (tibial cortical, tibial subchondral, femoral cortical, femoral subchondral) in the MMx knee as compared to control knee, indicating more porosity at all the regions, however the values were not statistically different from control knee (Figure 5C). The most affected ROI was femoral cortical (p, 0.07). Tb.Pf, measured at all the 4 ROIs (tibial cortical, tibial subchondral, femoral cortical, femoral subchondral) showed alterations in their values in MMx knee joint as compared to control counterparts, indicating a 4-D distortion in all the regions, however, these changes were not significantly different (Figure 5D). Tb.N, measured at 2 ROIs (tibial subchondral, femoral subchondral), were not different between MMx and control knee (Figure 5E) Cortical wall thickness was measured at all the 4 ROIs (tibial cortical, tibial subchondral, femoral cortical, femoral subchondral) in MMx knee joint, and their values did not differ significantly from the control knee. The most affected ROI was tibial cortical with $p$ value of 0.14 (Figure 5F).

\section{Histological assessment of knee sections: H\&E, Safranin O, and PSR staining: Collagen fibers evaluation}

H\&E staining of coronal sections from medial knee joint showed thinning and fibrillation of articular cartilage on the medial aspect of tibia in MMx knee (Figures 6B and 6B1), whereas, control knee showed no fibrillation (Figures $6 \mathrm{~A}$ and $6 \mathrm{~A} 1$ ). There was narrowing of space between femur and tibia in $\mathrm{MMx}$ group as compared to control group (Figure 6B vs. 6A). Chondrocytes were arranged in parallel rows in the deep zone of tibial articular cartilage in control knee (Figure 6A1), whereas, the parallel cellular arrangement was disrupted in the tibial articular cartilage of MMx knee (Figure 6B1). There was an increase in the number of hypertrophic chondrocytes in the calcified cartilage zone of articular cartilage in MMx knee (Figure 6B1) as compared to control knee (Figure 6A1). A non-osseous fibrotic tissue was present at the medial end of tibia in the MMx knee (Figure 6B1), however there was no osteophyte. Human OA knee cartilage, serving as positive control, showed H\&E staining (Figures 6C and 6C1). Decreased metachromasia and fibrillation is shown in human OA knee cartilage (Figures 6C and 6C1). In summary, H\&E staining showed that the less invasive MMx causes degenerative changes in articular cartilage of medial joint in rat. Articular cartilage from $\mathrm{MMx}$ and control knee stained positive with Safranin $\mathrm{O}$ for proteoglycans showing red coloration (Figures 7A and 7B). However, there was substantial loss of staining, dominantly in the medial aspect of tibia including fibrillation area, and in the femur of MMx knee (Figure 7B). Articular cartilage from human OA knee, serving as positive control, showed positive staining with safranin O. However, there was a loss of staining in the region of longitudinal separation of collagen bundles (Figure 7C) and fibrillation (Figure 7D) in the superficial layers of cartilage. Picrosirius red (PSR) staining exhibited red coloration in the articular cartilage of tibia and femur in medial control (Figure 8A) as well as in MMx (Figure 8C) knee joint under light microscopy. However, there was a reduction in the intensity of red coloration (i.e., decreased metachromasia), more in tibial cartilage and less in femoral cartilage in MMx knee (Figure $8 \mathrm{C} 1$ ) as compared to control knee (Figure 8A1). Articular cartilage from human OA knee, serving as positive control, stained positive for PSR (Figure $8 \mathrm{E}$ ) and also showed decreased metachromasia (Figure $8 \mathrm{~F}$ ) under light microscopy. In the control knee, the polarization light
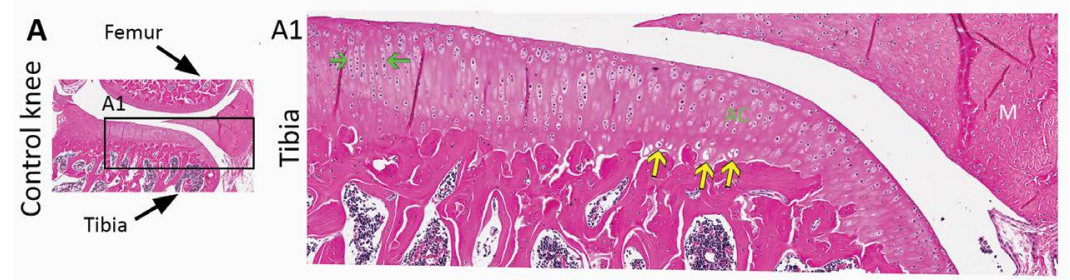

B

B1
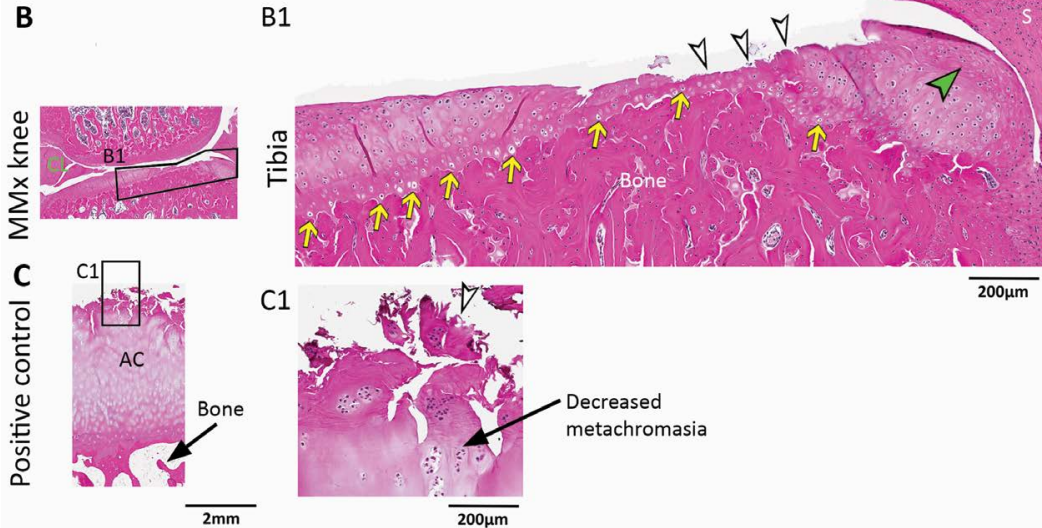

$200 \mu \mathrm{m}$

Human OA knee cartilage

(H \& E Staining)

Figure 6: H\&E staining of articular cartilage. Control (A), and MMx (B) knee joint. Human OA knee articular cartilage, served as positive control (C). A1, B1, and C1 are higher magnification of boxed area in $A, B$, and $C$, respectively. Note fibrillation in the articular cartilage (white arrowheads) and thinning of articular cartilage at the medial aspect of tibia, and the presence of a non-osseous fibrotic tissue (green arrowhead) at the medial end of tibia in MMx knee (B1). Chondrocytes are arranged in parallel rows (green arrows) in the tibial articular cartilage in control knee (A1), the parallel cellular arrangement disrupted in MMx knee (B1). Note an increased number of hypertrophic chondrocytes (yellow arrows) in the calcified cartilage zone in MMx knee (B1) as compared to control knee (A1). There is narrowing of space between tibia and femur in MMx knee joint as compared to control knee joint (B vs. A). Human OA knee cartilage, serving as a positive control, shows $H \& E$ staining (C, C1). Fibrillation (white arrowheads), and decreased metachromasia, is shown in human OA knee articular cartilage (C1). M, meniscus; S, synovium; CL, cruciate ligament; $A C$, articular cartilage. 
A

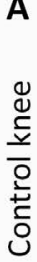

A

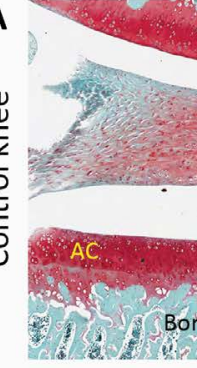

Horizontal separation

C
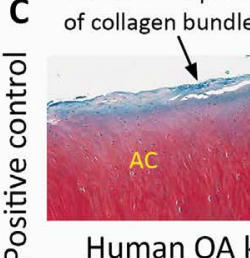

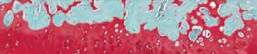

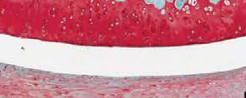

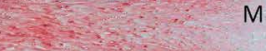

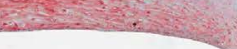
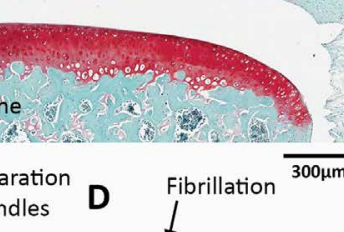

Fibrillation

$\overline{300 \mu \mathrm{m}}$

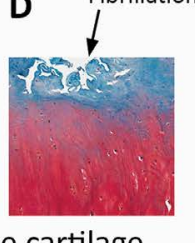

Human OA knee cartilage

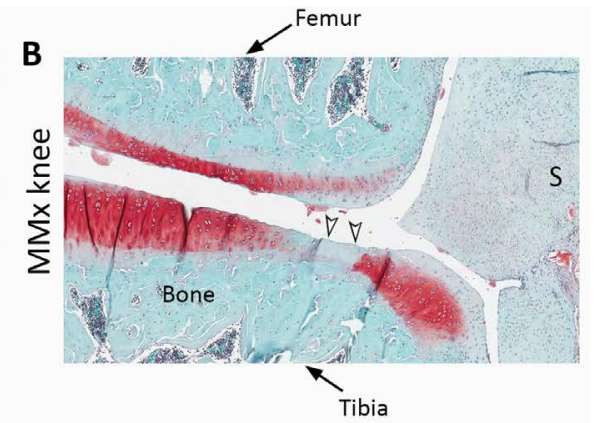

(Safranin O staining)

Figure 7: Safranin O staining in the articular cartilage. Control (A), and MMx (B) knee joint. Human OA knee articular cartilage, served as positive control (C, D). Articular cartilage from MMx knee as well as from control knee stained with Safranin O showing red coloration (8A, 8B). However, note the substantial loss of staining, dominantly in the medial aspect of tibia including fibrillation area (white arrowheads), and in the femur of MMx knee (8B). Articular cartilage from human OA knee shows positive staining with safranin $O$. However, there was a loss of staining in the region of longitudinal separation of collagen bundles (C) and fibrillation (D).

microscopy of mounted PSR-stained sections showed green coloration in superficial zone (indication of thin fibers) and the mixture of colors 'orange to red' (thick fibers) and green (thin fibers) indicating more thick and less thin 9 fibers in other part of the articular cartilage (Figures 8B-8B2a). In MMx knee, a large part of tibial and also a small part of femoral articular cartilage exhibited green coloration indicating the conversion of thick fibers into thin fibers in MMx knee articular cartilage (Figures 8D-D2a). Collagen fibers orientation, in fibrillated cartilage, was disrupted in MMx knee as compared to parallel collagen fibers arrangement in control knee (Figure 8D2a vs. 8B2a). Articular cartilage from human OA knee, serving as a positive control, showed mixture of colors but dominated by green color, indicating OA cartilage is rich in thin fibers (Figure $8 \mathrm{~F}$ ). Collagen fibers, in the surface area of human $\mathrm{OA}$ articular cartilage containing longitudinal collagen bundles, were disorganized and were not parallel to surface (Figures $8 \mathrm{~F}$ and $8 \mathrm{~F} 1$ ). In brief, the less invasive MMx caused alteration in collagen network and disruption of collagen fibers orientation in articular cartilage.

\section{Immunohistochemistry (IHC)}

Aggrecan expressed in the articular cartilage of control and MMx knee (Figures 9A and 9B). However, aggrecan staining was decreased in the articular cartilage of MMx knee as compared to control knee (Figure 9B vs. 9A). Higher magnification of tibia shows that reduction or loss of aggrecan staining was dominant in the fibrillation area (red arrowheads) as compared to other part of the tibia in MMx knee (Figure 9B1). Human OA knee articular cartilage, serving as positive control, showed areas where aggrecan was expressed (Figures 9C and 9C1) but also showed damaged areas where aggrecan was absent (Figure 9C, red arrows). Note that aggrecan showed territorial and inter-territorial expression in control articular cartilage (Figure 9A1), whereas, expression was more territorial in $\mathrm{MMx}$ knee articular cartilage (Figure 9B1), and human OA knee articular cartilage as well (Figure 9C1). Cleaved aggrecan neoepitope, G1 NITEGE374 (NITEGE) expressed in the articular cartilage of control knee (Figures
9D-9D2) and MMx knee (Figures 9E-9E2). However, the intensity of NITEGE expression was higher in all areas of articular cartilage except the fibrillated area where there was less aggrecan available (Figures 9E-9E2). Human OA knee articular cartilage, serving as a positive control, showed NITEGE staining (Figures 9F and 9F1). COLII showed expression in the articular cartilage of control and $\mathrm{MMx}$ knee (Figures 10A and 10B). The staining in MMx knee was reduced (Figures 10B and 10B1) as compared to control knee (Figures 10A and 10A1). Articular cartilage, in human OA knee (Figures 10C-10C2) and in human normal trachea (Figure 10D) serving as positive controls, showed COL-II expression. Denatured COL-II fragment 3/4 (-2-3/4 $\mathrm{m})$ expressed at higher level in the articular cartilage of MMx knee as compared to control knee (Figures 10F1 and 10F2 vs. 10E1 and 10E2). As a positive control, human OA knee articular cartilage showed intense staining for Col2-3/4m (Figures 10G-10G2), the intensity varied from fibrillated to non-fibrillated region, and from superficial zone to deep zone (Figures 10G-10G2). COL-X expression was low, and restricted to hypertrophic chondrocytes near the tidemark in the articular cartilage, in control rat knee (Figures 11A-11A2). However, the 10 intensity of COL-X expression increased around tidemark and the in calcified cartilage zone; the expression further extended to non-calcified deep zone (Figures 11B1 and 11B2). P1 mouse spinal vertebrae, serving as positive control, showed COL-X expression in the hypertrophic chondrocytes (Figures 11C and 11C1). MMP13 expressed in the tangential, transitional, radial, and calcified cartilage zone of the articular cartilage with variation in intensities among the zones in the control knee (Figures 12A-12A2), as well as in the MMx knee (Figures 12B-12B2). The expression level was higher in the articular cartilage of MMx knee as compared to control knee (Figures 12B1 and $12 \mathrm{~B} 2$ vs. $12 \mathrm{~A} 1$ and $12 \mathrm{~A} 2$ ). Human OA knee articular cartilage, serving as positive control, showed MMP13 expression, in all the zones but with variation in the intensity among the zones (Figures 12C-12C3). Lubricin (PRG4) expressed in the superficial zone of articular cartilage in the control knee (Figures 13A-13A2). The expression was also present in the articular cartilage in MMx knee (Figures 13B-13B2) except in 
Citation: Juneja SC, Ventura M, Jay GD, Veillette C (2016) A Less Invasive Approach of Medial Meniscectomy in Rat: A Model to Target Early or Less Severe Human Osteoarthritis. J Arthritis 5: 193. doi:10.4172/2167-7921.1000193
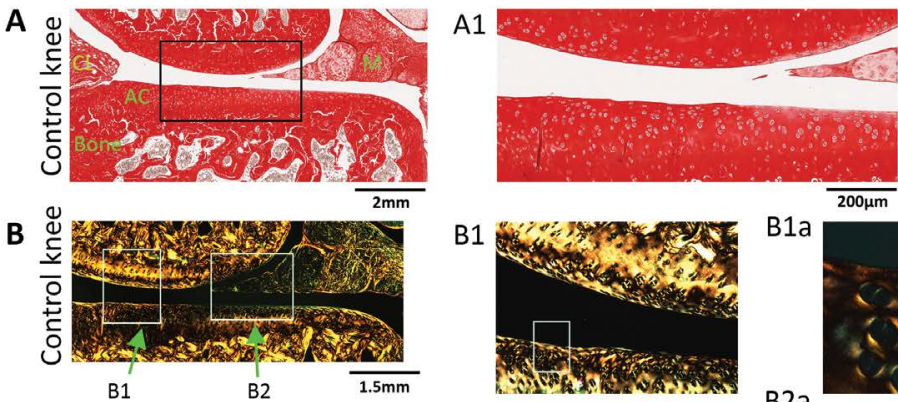

B2
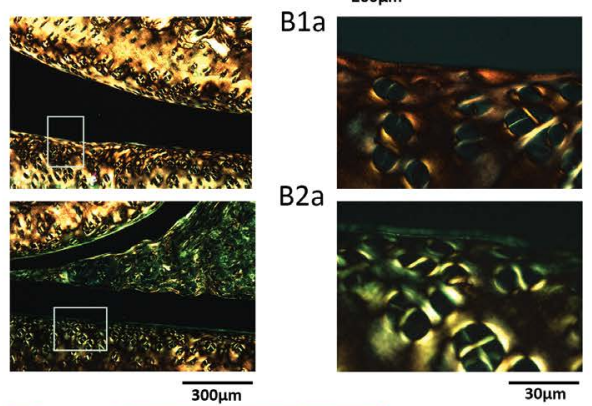

$\mathrm{B} 2 \mathrm{a}$
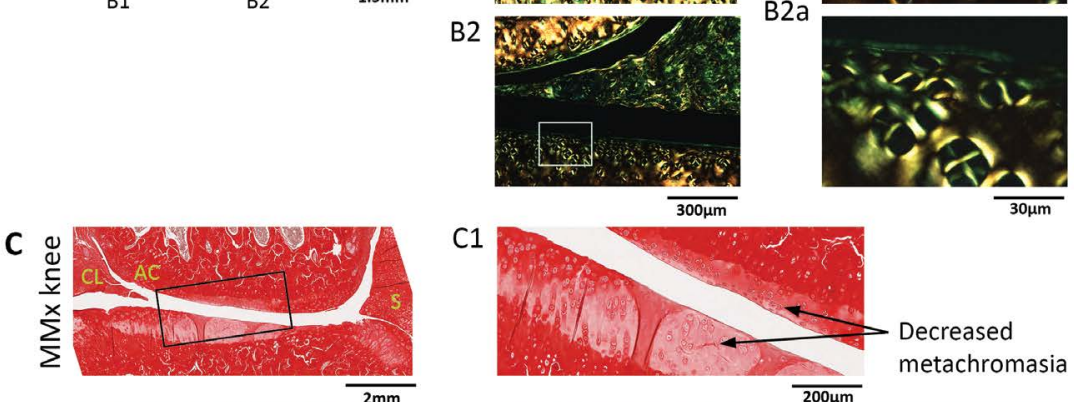

C1
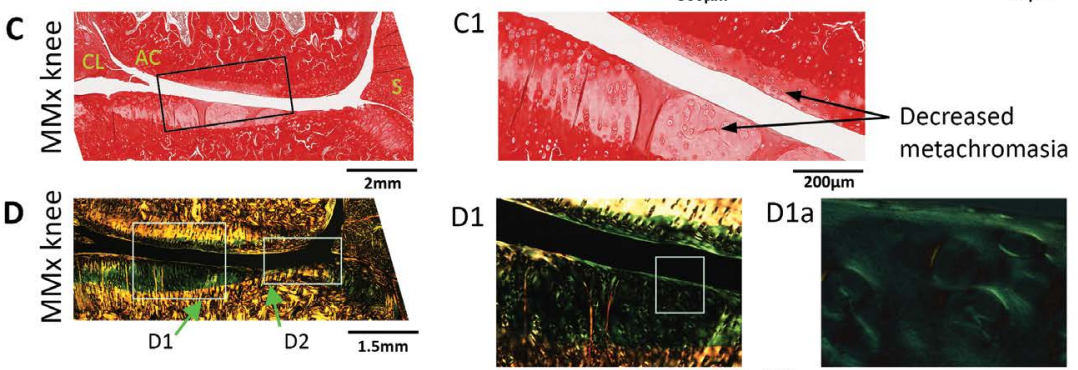

D2
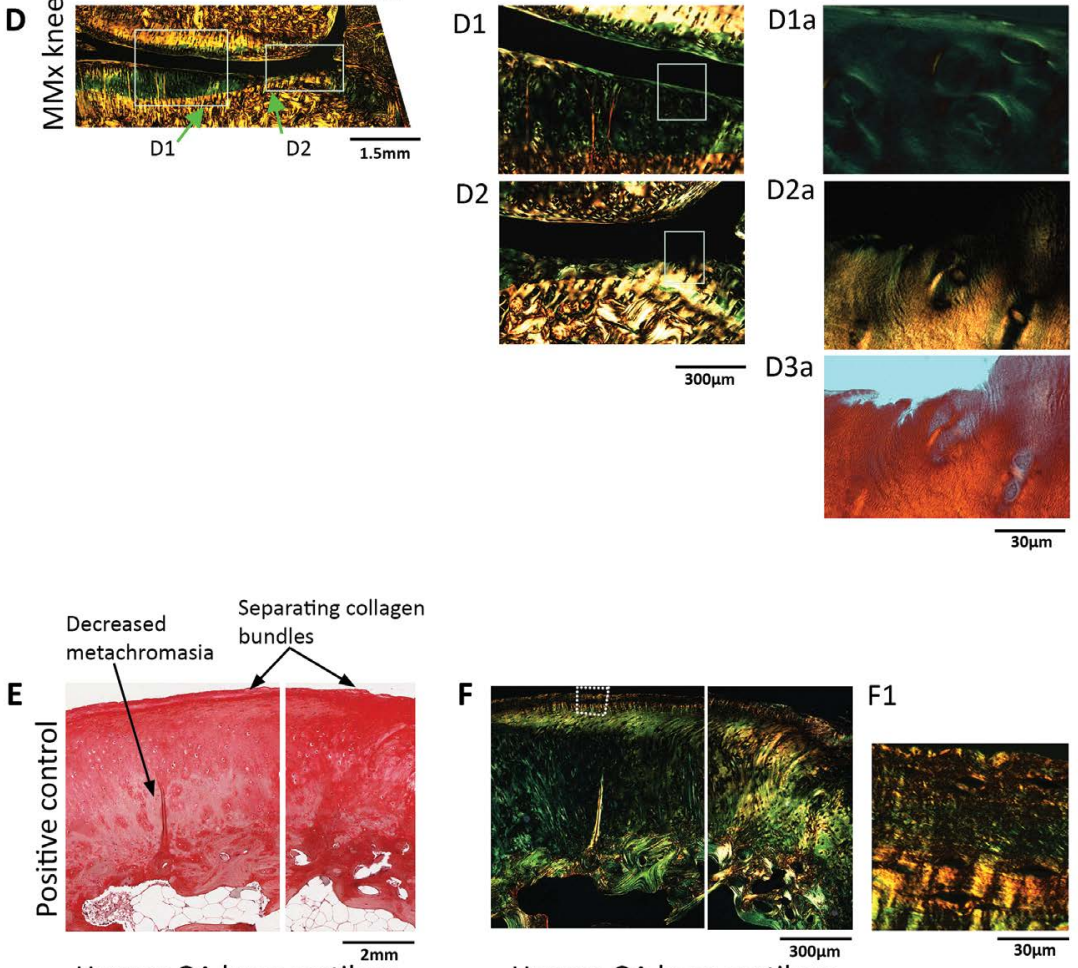

F1

Human OA knee cartilage

Human OA knee cartilage

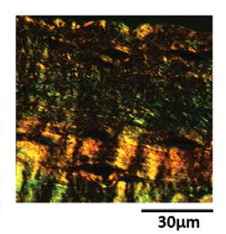

(Picrosirius red staining)

Figure 8: Picrosirius red (PSR) staining. Light microscopy and polarization light microscopy of control and MMx rat knee joint (A-D). Light microscopy of PSR-stained coronal sections of control (A), and MMx (C) knee joint. Polarization light microscopy of PSR-stained sections of control (B) and MMx (D) knee joint. PSR-stained human OA knee articular cartilage, served as positive control for light microscopy (E) and for polarization light microscopy (F). A1, B1/B2, C1 and D1/D2 are higher magnification of boxed area in A, B, C and D, respectively. B1a, B2a, D1a, D2a and F1 are higher magnification of boxed area in B1, B2, D1, D2 and F, respectively. Key observations noted are described as below: $\$$ Decreased intensity of PSR staining (i.e., decreased metachromasia) in the articular cartilage of MMx knee as compared to control knee (C1 vs. A1) is shown; \$ More thick collagen fibers (orange-red colored) and less thin collagen fibers (green colored) present in the articular cartilage of control knee (B), on the other hand, more thin collagen fibers (green colored) and less thick collagen fibers (orange-red colored) present in the articular cartilage of MMx Knee (D); § At higher magnification, it is more clear that more thin collagen fibers (green colored) in MMx knee articular cartilage (D1a) as compared to control knee articular cartilage (B1a, B2a) are present; $\mathrm{D} 2 \mathrm{a}(\mathrm{MMx})$ being fibrillated, most of collagen fibers are missing, cannot be compared with non-fibrillated region of $\mathrm{B} 2 \mathrm{a}$ (control knee); $\S$ Collagen fiber orientation of surface zone of D2a (MMx) is impaired as compared to that of B2a (control); Fibrillation area (D3a) showing under light microscopy $\$$ Decreased metachromasia in PSR-stained human OA knee articular cartilage (E); Human OA knee articular cartilage (F1) contains mainly thin collagen fibers (green coloration) under polarization light; $\S$ Surface of human OA articular cartilage contains longitudinal separation of collagen bundles. Surface collagen fibers are not aligned parallel; rather collagen fibers are disoriented (F1). 
Citation: Juneja SC, Ventura M, Jay GD, Veillette C (2016) A Less Invasive Approach of Medial Meniscectomy in Rat: A Model to Target Early or Less Severe Human Osteoarthritis. J Arthritis 5: 193. doi:10.4172/2167-7921.1000193
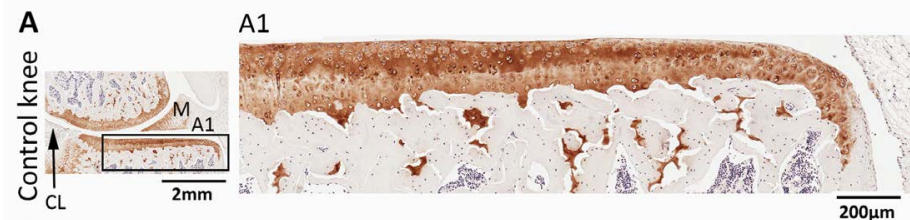

B

B1 $\overline{200 \mu \mathrm{m}}$
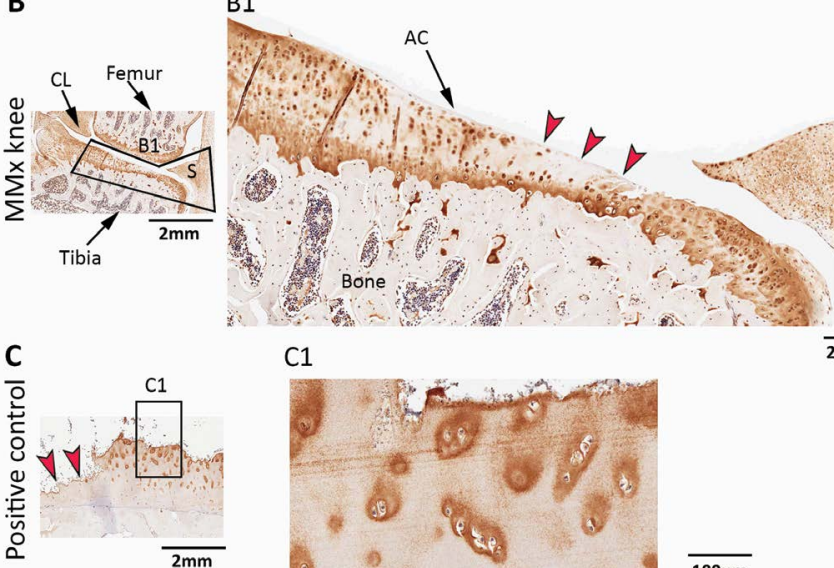

C1

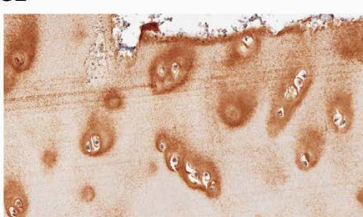

$\overline{200 \mu m}$

Human OA knee cartilage
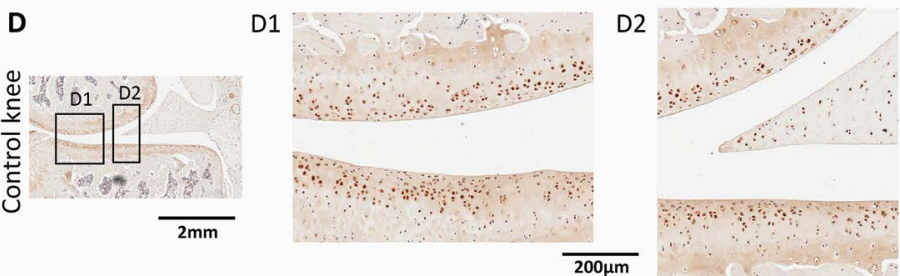

E

E1
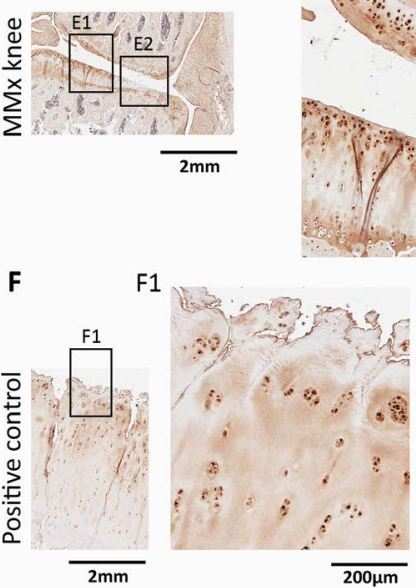

Human OA knee cartilage

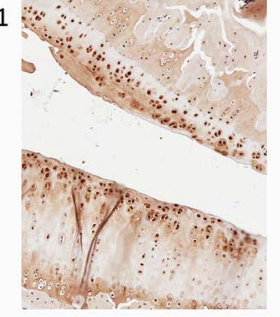

E2

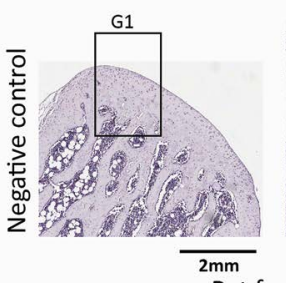

G1

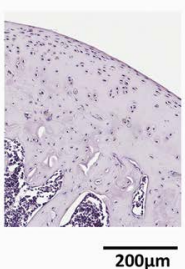

Figure 9: IHC of aggrecan the articular cartilage. Control (A), and MMx (B) knee joint. Human OA knee articular cartilage, served as positive control for aggrecan. $A 1, B 1$, and $C 1$ are higher magnification of boxed area in A, B and C, respectively. Aggrecan expressed in the articular cartilage of control and MMx knee (A and B). However, aggrecan staining was decreased in the articular cartilage of MMx knee as compared to control knee (B vs. A). Higher magnification of tibia shows that reduction or loss of aggrecan staining was dominant in the fibrillation area (red arrowheads) as compared to other part of the tibia in MMx knee (B1). Human OA knee articular cartilage, serving as positive control, showed areas where aggrecan was expressed (C. C1) but also shows damaged areas where aggrecan was absent (C, red arrows). Note that aggrecan showed territorial and inter-territorial expression in control articular cartilage (A1), whereas, expression was more territorial in MMx knee articular cartilage (B1) and human OA knee articular cartilage (C1). IHC of NITEGE in the articular cartilage. Cleaved aggrecan neoepitope, G1 NITEGE374 (NITEGE) IHC in control (D), and MMx (E) knee joint. Human OA knee articular cartilage, served as positive control for NITEGE (F). Rat femoral articular cartilage, served as negative control (G). D1/D2, E1/E2, F1 and G1 are higher magnification of boxed area in D, E, F and G, respectively. NITEGE expressed in the articular cartilage of control knee (D, D1, D2) and MMx knee (E, E1, E2). However, the intensity of NITEGE expression was higher in all areas of articular cartilage except the fibrillated area where there was no aggrecan available (E, E1, E2). Human OA knee articular cartilage, serving as a positive control, showed NITEGE staining (F, F1). Rat femoral articular cartilage, serving as negative control for aggrecan and NITEGE, showed no staining in the articular cartilage (G, G1). 
A

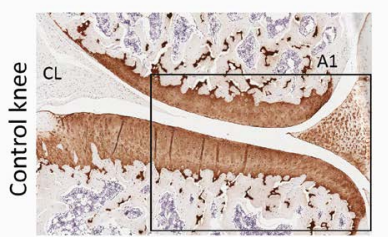

B

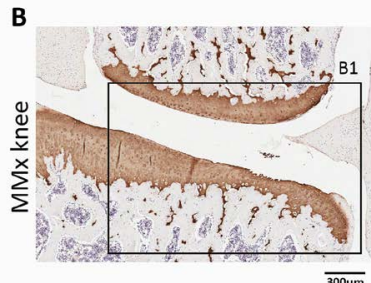

A1

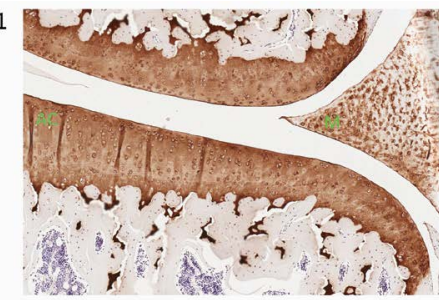

B1

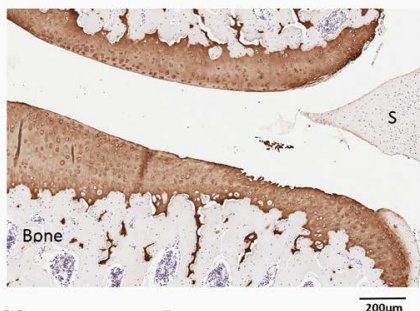

$\mathrm{C} 2$

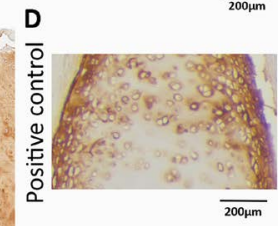

Human normal

tracheal cartilage
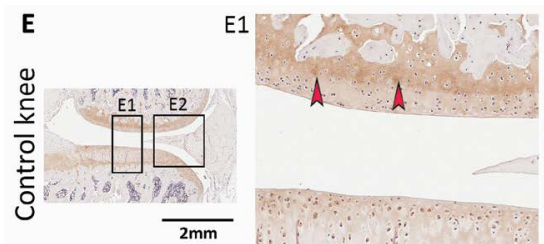

E2

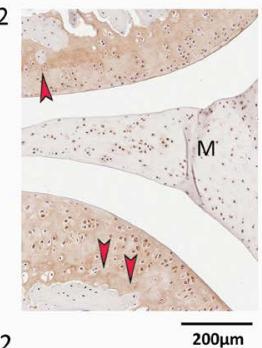

F1
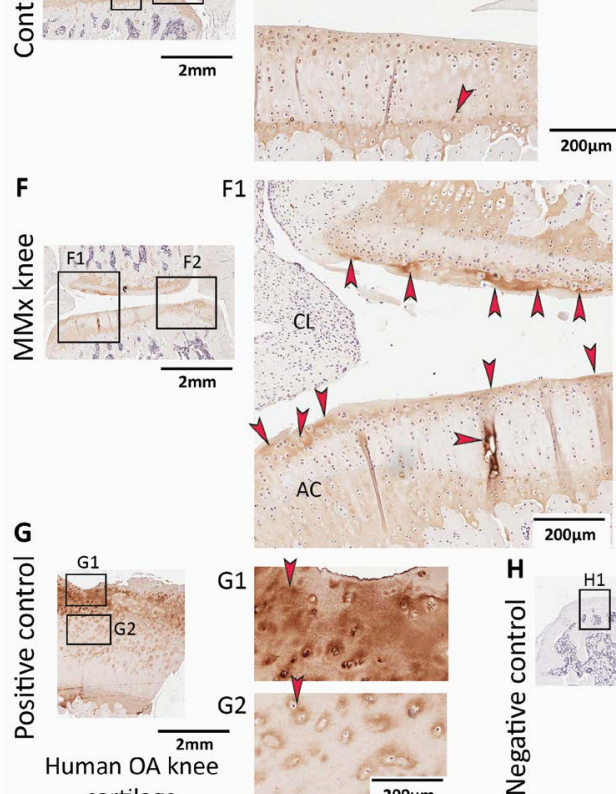

cartilage

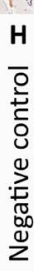

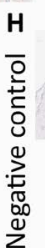
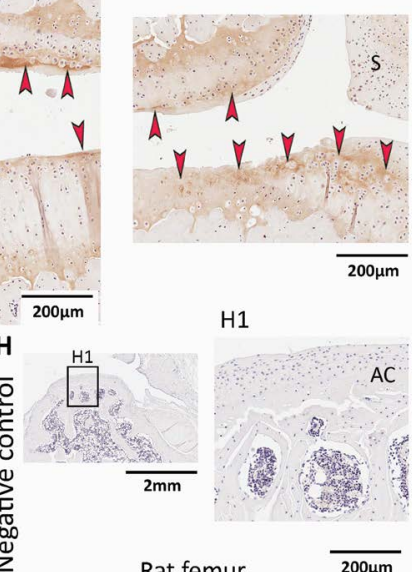

$\mathrm{H}$

Figure 10: IHC of COL-II in the articular cartilage. Control (A), and MMx (B) knee joint. Human OA knee articular cartilage, and human normal tracheal cartilage, served as positive control (C), and rat femur articular cartilage, as negative control (H). A1, B1, C1/C2 are higher magnification of boxed area in A, B, and C, respectively. Articular cartilage showed reduced immunostaining for COL-II in MMx as compared to control knee (B1 vs. A1). Human OA knee articular cartilage, serving as positive control, show reduced positive staining for COL-II (C2) and contained area where staining was lacking (red arrowheads, C1). Human normal tracheal cartilage showed intense COL-II immunostaining (D). IHC of Col2-3/4 in the articular cartilage. Denatured COL-II fragment $3 / 4$ (Col2-3/4 m) in the articular cartilage of control $(\mathrm{E})$, and $\mathrm{MMx}(\mathrm{F})$ knee joint. Human OA knee articular cartilage, served as positive control (G), and rat femur articular cartilage, as negative control (H). E1/E2, F1/F2, G1/G2, H1 are higher magnification of boxed area in E, F, G and H, respectively. Col2- 3/4m expression was higher in the articular cartilage of MMx knee as compared to control knee (red arrowheads; F1, F2 vs. E1, E2). As a positive control, human OA knee cartilage showed intense staining for Col2-3/4 m (G, G1, G2), the intensity varied from fibrillated to non-fibrillated region, from superficial zone to deep zone (G, G1, G2). Rat femur, serving as a negative control for COL-II and Col-2/3m exhibited no staining $(\mathrm{H}, \mathrm{H} 1)$. 
Citation: Juneja SC, Ventura M, Jay GD, Veillette C (2016) A Less Invasive Approach of Medial Meniscectomy in Rat: A Model to Target Early or Less Severe Human Osteoarthritis. J Arthritis 5: 193. doi:10.4172/2167-7921.1000193

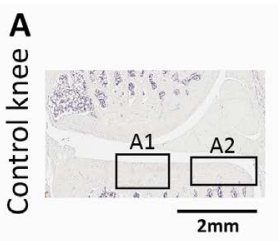

A1 A2
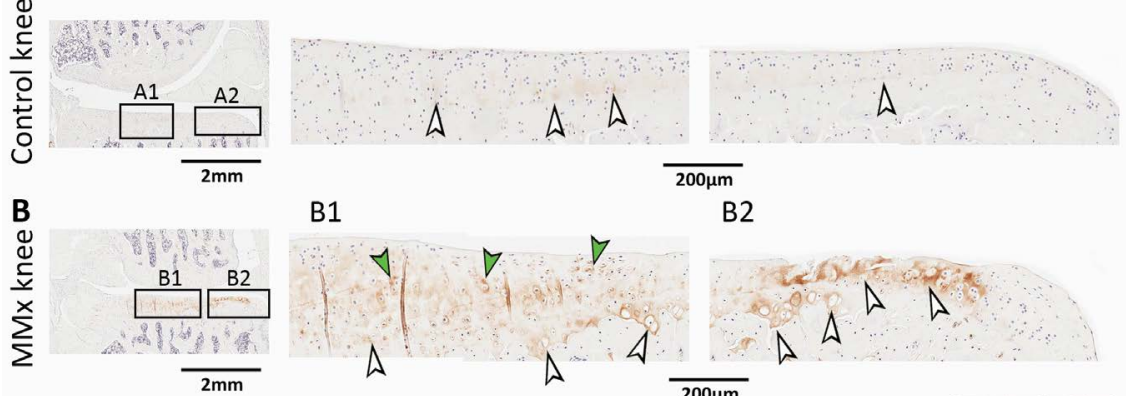

B1

B2
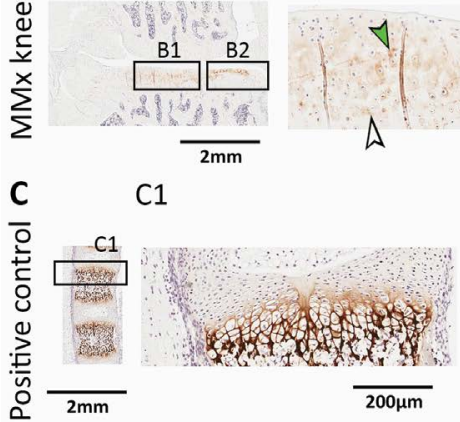

P1 mouse spine

Rat femur

$\Delta A$

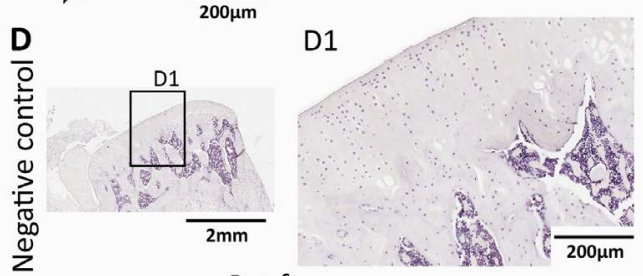

Figure 11: IHC of COL-X in the articular cartilage. Control (A), and MMx (B) knee joint. Mouse P1 spine, served as positive control (C). Rat femoral articular cartilage, served as negative control (D). A1/A2, B1/B2, C1 and D1 are higher magnification of boxed area in A, B, C and D, respectively. COL-X expressed weakly near tidemark (white arrowheads) dominantly in the tibial articular cartilage (A1, A2). However, the intensity of COL-X expression increased around tidemark and in calcified cartilage zone (white arrowheads); and also the expression extended to non-calcified deep zone (green arrowheads, B1, B2). P1 mouse spinal vertebrae, serving as positive control, showed COL-X expression in hypertrophic chondrocytes (C, C1). Rat femur articular cartilage, serving as negative control, exhibited no staining (D, D1).

\section{A}

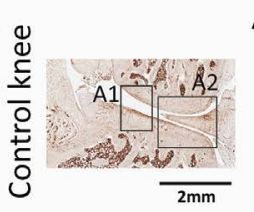

B

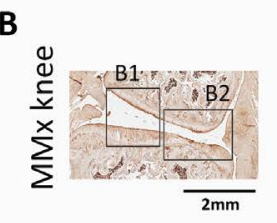

MMP13 expression in different zones of articular cartilage: Tangential (white arrowhead); Transitional arrowhead); Calcified (red arrowhead)

C

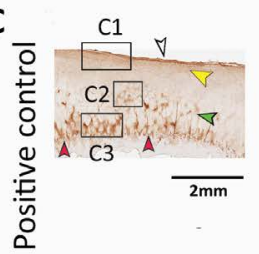

A1

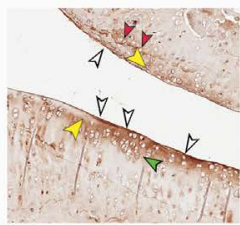

B1

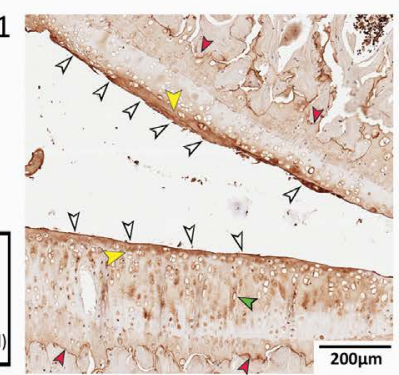

A2

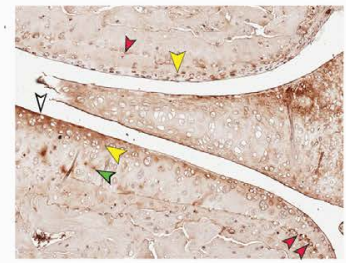

B2

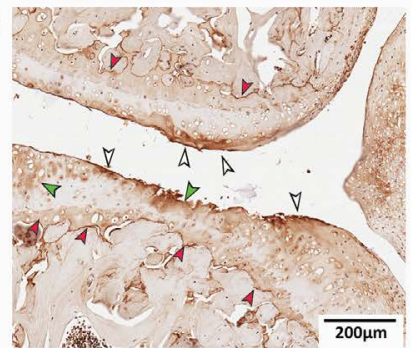

C1

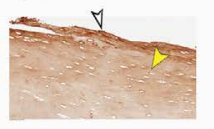

C3

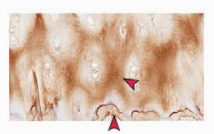

Human OA knee cartilage

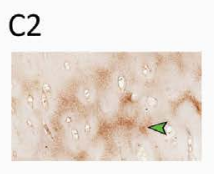

$\overline{200 \mu m}$

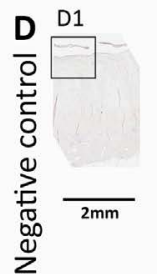

Human OA knee cartilage
D1

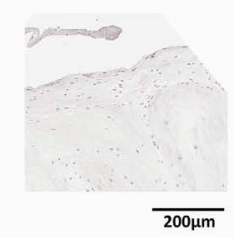

Figure 12: IHC of matrix metallopeptidase 13 (MMP13) in the articular cartilage. Control (A), and MMx (B) knee joint. Human OA knee articular cartilage, served as positive control (C) and as negative control (D). A1/A2, B1/B2, C1/C2/C3 and D1 are higher magnification of boxed area in A, B, C and D, respectively. MMP13 expressed in the tangential zone (white arrowhead), transitional zone (yellow arrowhead), radial zone (green arrowhead), and calcified cartilage zone (red arrowhead) of articular cartilage in the control knee (A, A1, A2) as well as in the MMx knee (B, B1, B2). The expression level was higher in the articular cartilage of MMx knee as compared to control knee (B1, B2 vs. A1, A2). Human OA knee articular cartilage, serving as positive control, showed MMP13 expression, in all the zones with variation in the intensity among the zones (C, C1, C2, C3). Human OA knee articular cartilage, serving as negative control, did not show staining (D, D1). 
the fibrillated region at the medial aspect of tibia where superficial/ transitional zone and part of radial zone was damaged in the articular cartilage (Figure 13B2). Human OA knee articularcartilage, serving as positive control, showed lubricin expression in the superficial zone and transitional zone (Figures 13C-13C2).

\section{Discussion}

\section{Less invasive $M M x$ causes non-significant changes in subchondral bone in medial knee joint}

Animal models of OA are tools for investigating the development of the disease more quickly than human develops OA in real life. Mice or rats are particularly of great interest because of their small size, short life span, breeding efficiency and commercial availability. A number of genetically altered mice are also available at an increasing rate and are being used as surgically-induced OA models that provides a great deal of information on the genes playing role in OA [28]. MMx-induced knee $\mathrm{OA}$ is a common experimental procedure in mice and rats $[15,21,22]$ that is to get the first-hand information on the effectiveness of therapeutical agent before testing it on large animals and or in human trials. MMx surgery conducted, on medial knee capsule, involved the following invasive steps, i.e., beginning with transecting
MCL followed by dislodging the patella from the trochlear groove and diverting it laterally and exposing the knee capsule wide-open, and finally transecting the medial meniscus at entheses attachments $[15,21,22]$. Following the procedure that involved wide opening of knee capsule for MMx, substantial subchondral bone degeneration has been reported in MMx rat knee at 8-weeks post-MMx, as determined by $\mu \mathrm{CT}$ analysis [23]. Using a joint injury to initiate joint degeneration represents post-traumatic OA. 'Less invasion' to 'more invasion' to cause post-traumatic OA may have significant effects due to the surgical/invasive injury procedure itself, rather than the targeted joint injury [29]. We took a less invasive approach on MMx surgery in the rat. By using eye loupes (4.9x), it was easy to see the location of medial meniscus on medial aspect of the intact knee capsule. Therefore it was easy to avoid transection of MCL, and MMx surgery was conducted with minimum damage to knee capsule without exposing the knee capsule wide-open (Figure 1) and thus causing minimum synovial inflammation in the knee capsule. Synovial inflammation has directly been linked to enhance OA [26]. The major part of meniscus including wide portion was cut removed in our procedure that was responsible for degenerative changes in articular cartilage and also that was equivalent to standard MMx or DMM procedure that causes articular cartilage degeneration as well as subchondral bone degenerative
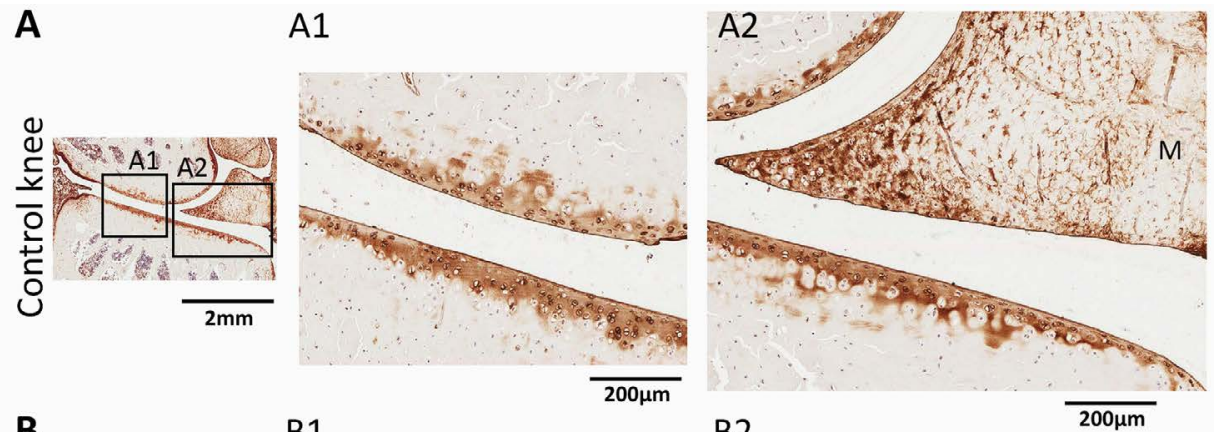

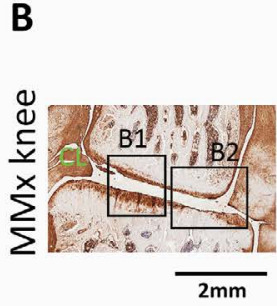

B1
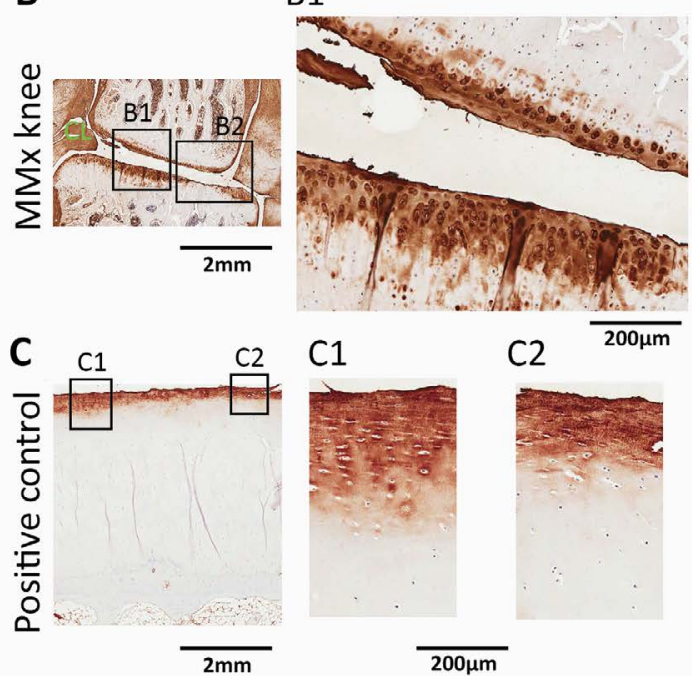

C1

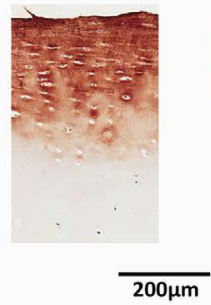

C2

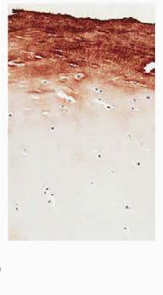

Human OA knee cartilage
B2

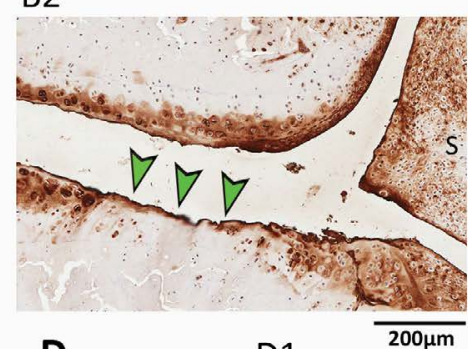

D

D1

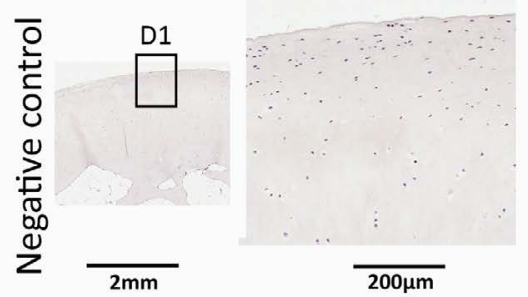

Human OA knee cartilage

Figure 13: IHC of lubricin (PRG4) in the articular cartilage. Control (A), and MMx (B) knee joint. Human OA knee articular cartilage, served as positive control (C) and as negative control (D). A1/A2, B1/B2, C1/C2 and D1 are higher magnification of boxed area in $A, B, C$ and D, respectively. Lubricin showed expression mainly in the superficial zone of articular cartilage in the control knee (A, A1, A2). However, in MMx knee, lubricin expression was present in the articular cartilage except in the fibrillation area (arrowheads) at the medial aspect of tibia where superficial/transitional zone and part of deep zone of articular cartilage is damaged. Human OA knee articular cartilage, serving as positive control, shows lubricin expression in the superficial zone and transitional zone (C, C1, C2). Human OA knee articular cartilage, serving as negative control, did not show staining (D, D1) 
changes (23). Osteophytes and subchondral sclerotic changes were reported in mice, at 8 weeks post-surgery for knee instability, imaged at subluxation position by X-ray radiography [30]. We imaged knee joints at different views (PA view of whole body; ML, AP and PA view of knee joint; Figures 2A-2D). We detected no noticeable sized osteophyte at the medial margin of MMx tibia. And also there was no bone sclerosis in subchondral bone region (Figures 2A-2D). X-ray imaging showed that the current less invasive MMx procedure did not cause any subchondral bone degeneration in rat. As shown by $\mu \mathrm{CT}$ images of knee joint, the MMx procedure was successful in this investigation (Figures $4 \mathrm{~A}$ and $4 \mathrm{~B}$ ). Bone strength parameters (i.e., BV/TV, Tb.Th, Tb.Sp and Tb.N etc.) have been shown to measure bone strength alterations with age, pharmaceutical intervention or gene mutation [31-34]. Subchondral bone strength parameters changed after OA induction [35]. In an elegant study of collagenase-injected-induced arthritis (CIA) model of mice, subchondral bone damage was shown by significant changes in bone strength parameters (BV/TV, Tb.Sp and $\mathrm{Tb} . \mathrm{Th}$ ) that occurred upto 14 weeks post-treatment [16]. Using similar bone parameters, to measure the subchondral bone strength in a less invasive MMx rat, we discovered that there were no significant changes in the values of bone parameters (BV/TV, Tb.Th, Tb.N, Tb.Sp, Tb.Pf, cortical wall thickness) in tibial and femoral cortical or tibial and femoral subchondral ROIs (Figure 5). In another study of monosodium iodoacetate (MIA)-induced OA model of mice, MIA injection in knee resulted in a significant decrease in BV/TV and an increase in Tb.Sp but with no changes in Tb.N in rats after 5-days of post-treatment [36]. Panahifar and colleagues showed substantial degenerative changes in subchondral bone in rat knee at 8 weeks post-MMx, as determined by $\mu \mathrm{CT}$, along with articular cartilage degenerative changes [23]. The current study demonstrated that the less invasive MMx procedure caused no changes in subchondral bone.

\section{Less invasive MMx causes articular cartilage degeneration at moderate OA level}

Histological evaluation by H\&E staining, proteoglycans, and collagen localization: Fibrillation and thinning of articular cartilage, in the knee joint, has been shown in experimentally induced OA in several species including rat and mouse $[15,21,22]$, and human OA. The narrowing of space is a common feature in OA [2]. Our study showed that the less invasive MMx procedure, in medial rat knee joint, causes thinning and fibrillation of articular cartilage at the medial aspect of medial tibial articular cartilage, and narrowing of medial knee joint (Figures 6B and 6B1). The parallel arrangement of chondrocyte rows has been shown in the knee articular cartilage, and the disruption of the parallel arrangement has been linked to OA [37]. Our study showed that less invasive MMx procedure in rat, caused disruption of parallel arrangement of chondrocyte rows (Figure $6 \mathrm{~A} 1$ vs. $6 \mathrm{~B} 1$ ). We also showed the presence of non-osseous fibrotic tissue at the medial end of tibia (Figure 6B1). In OA joints, periarticular fibrotic deposits have been shown near erosion sites in OA in mice [38]. Our study showed that the less invasive MMx causes an increase in the hypertrophic chondrocytes in calcified cartilage zone in medial tibia (6B1) similar to report by Hayami and team in ACLT, and ACLT with MMx rats [12]. In brief, the less invasive $\mathrm{MMx}$ procedure, in rat knee joint, caused a number of degenerative 12 changes in the articular cartilage. Safranin O staining detects proteoglycans (PGs) in the articular cartilage [39]. Earlier studies indicated that the human knee articular cartilage from OA patients exhibited reduction in PGs asshown by reduced safranin O staining [40]. And similar findings are available in animal models. PGs showed reduction in articular cartilage of OA knee joints in 8 weeks post-MMx rats [41], In the current study, by using safranin
O staining, we showed that there was substantial loss of PGs in the articular cartilage dominantly in the medial aspect of knee joint (Figure 7B). Mild and severe OA cartilage, in human patients, showed the absence of PGs in the area closer to superficial zone [40]. In the current study, we demonstrated that the surgically induced less invasive MMx procedure that was effective in causing degenerative changes in the articular cartilage of rat knee.

Picrosirius red (F3BA) is a linear anionic dye comprising six sulfonate groups that can associate with cationic collagen fibers, and enhance their birefringence under cross-polarized light [42-44]. The collagen fibers in the ECM can be classified as thin fibers $(\leq 0.8 \mu \mathrm{m})$ or as thick fibers (1.6 to $2.4 \mu \mathrm{m}$ ) [44]. Integrity and organization of the collagen fibers represents the repair activity of articular cartilage $[45,46]$. Biomechanical models have shown that the changes in collagen network influence the biomechanical behavior of the cartilage [47]. The current study showed decrease in PSR staining or metachromasia in the articular cartilage of MMx knee, indicating alteration in collagen network (Figures $8 \mathrm{C}$ and $8 \mathrm{C} 1$ ). Polarization light microscopy of PSRstained cartilage revealed decrease in birefringence intensity in the superficial-middle zones of patellar cartilage of laterally meniscectomized joints in rat [48]. In the current study, MMx caused thinning of collagen fibers in articular cartilage in rat knee as shown by dominance of green coloration under polarization light (Figures $8 \mathrm{D}-8 \mathrm{D} 2)$. In brief, the study indicates that the less invasive MMx was effective in causing alteration in collagen fibril network in the articular cartilage. In human OA cartilage, green colored thin fibers were dominating (Figure 8F). Collagen fiber orientation was altered in fibrillated surface region of articular cartilage in MMx knee as compared to control non-fibrillated cartilage, the later being parallel to surface (Figure 8D2a vs. 8B2a). Human OA knee cartilage showed disoriented collagen fibers orientation (Figure 8F1).

\section{Less invasive $M M x$ causes alteration in articular cartilage markers}

Aggrecan, and cleaved aggrecan neoepitope (NITEGE): Aggrecan is the major proteoglycan of hyaline cartilage and it associates with hyaluronic acid (HA) and link protein, to form aggregates [49]. The multi-molecular structures can contain $>100$ molecules of aggrecan and link protein associated with a single HA chain, that give rise to aggregates. The aggregates are hydrated due to their negative charge resulting from the large number of poly-anionic GAG chains on aggrecan, providing cartilage with a high water content about $70 \%$ wet weight of the tissue [50]. They act as a space-filling gel and are responsible for the compression and resilience of articular cartilage during joint loading. A mutation in the variable repeat region of the aggrecan gene causes a form of spondyloepiphyseal dysplasia (SEDC) associated with severe premature OA [51]. In knee joint articular cartilage, aggrecan expression was shown to be present in calcified cartilage and non-calcified cartilage zone. Aggrecan contributes to territorial and inter-territorial matrix in mice [52]. In the current study, MMx causes reduced expression of aggrecan in the knee tibial articular cartilage, indicating that the less invasive MMx procedure was effective in causing degenerative changes in the articular cartilage in the medial compartment of the knee (Figure 9B vs. 9A). Suppression of anabolic activity of chondrocytes in the upper zones contributes to the metabolic imbalance observed in OA cartilage [53]. Using ISH, most chondrocytes in the deeper zones of OA cartilage showed an increase in aggrecan mRNA expression, as compared with normal specimens. However, chondrocytes of the upper zone were largely negative for aggrecan mRNA [53]. More loss of aggrecan is shown in medial aspect 
of tibial cartilage since that region represents non-physiological contact zone between femur and tibia and that causes fibrillation and some loss of chondrocytes and matrix (Figure 9B1). Aggrecan is composed of three major globular domains: G1, G2, and G3. Between G1 and G2 domains, there is an interlobular domain (IGD) that is the major site of cleavage by specific proteases like MMPs and aggrecanase (ADAMTS) [54]. Aggrecanase cleaves aggrecan in the IGD at Glu373-Ala374, generating a G1 fragment with a $\mathrm{COOH}$ terminus of NITEGE373. The cleaved G1 fragment NITEGE373 can be recognized by an antibody called NITEGE [55], the expression of which increased with age in articular cartilage [56]. In a CIA rat model of cartilage degeneration, NITEGE neoepitope increased on day 21 post-injection [57]. The current study showed that the less invasive MMx procedure, in rat the knee, causes degradation of aggrecan as shown by an increase in NITEGE immunostaining in knee articular cartilage (Figures 9E1 and 9E2) except fibrillated region, the later being the damaged area lacked aggrecan to begin with (Figure 9B1).

Type II collagen (COL-II), and Denatured COL-II 3/4 fragment (Col2-3/4m): COL-II is specific for cartilaginous tissues. It is essential for normal embryonic development of the skeleton, linear growth, and for the ability of cartilage to resist compressive forces. The major reason of OA is the degradation of hyaline articular cartilage [58]. Mutation in COL2A1 gene results in abnormal cartilage morphology and bone development. With a single point substitution mutation in COL2A1 in families (Arg75Cys), the kindred displayed early onset OA and mild spondyloepiphyseal dysplasia [59]. MMx caused reduced COLII expression in rat knee articular cartilage (Figures 10B and 10B1) indicating that the less invasive MMx procedure was effective in causing degenerative changes in the articular cartilage. Kaufman and team reported that ACLT caused significant loss of COL-II immunostaining in articular cartilage surface in rat [60]. In one study, $\mathrm{Xu}$ and colleagues showed reduction in COL-II immunostaining in the articular cartilage by more than $60 \%$ at 6 weeks post-surgery involving ACLT and MMx [61]. Interstitial collagenases (collagenase-1, -2, and -3) catalyze specific cleavage site of collagen at the intra-helical 775Gly-776Ile bond [62] This generates characteristic three-quarter $(3 / 4)$ and one-quarter $(1 / 4)$ collagen fragments. These fragments are spontaneously denatured and then rapidly degraded by collagenases and gelatinases [63]. Col2-3/4c antibody can detect native form of cleaved Col2-3/4 fragment, whereas, Col2-3/4m antibody can detect degraded form of the same fragment, by immunoassays. We demonstrated the expression of denatured Col2$3 / 4$ fragment in less invasive MMx knee articular cartilage in the medial knee joint by using Col2-3/4m antibody, and that indicated increased COL-II degradation in the articular cartilage (Figures 10F-10F2). On the other hand, control rat knee showed lower immunostaining for Col2- 3/4m (Figures 10E-10E2). The immunostaining clearly demonstrates that less invasive MMx causes degenerative changes in articular cartilage.

Type $\mathbf{X}$ collagen (COL-X): The synthesis of COL-X is mostly restricted to hypertrophic chondrocytes, regardless of whether these cells are found in growth plate or OA cartilage [64,65]. Mutations in COL10A1 gene has been shown to lead to Schmid metaphyseal chondrodysplasia, a rare autosomal dominant disorder of the skeleton that is manifested in early childhood by short stature, coxa vara and a waddling gait [66]. COL-X mutant mice develop coxa vara, exhibit reduction in thickness of growth plate resting zone cartilage and articular cartilage, and altered bone content [67]. In the current study, the less invasive MMx caused an increase in COL-X expression near tidemark, and in the calcified cartilage zone, and also caused induction of expression in the deep zone of articular cartilage (Figures 11B1 and
11B2). Similar findings have been reported in a study in which ACL was injured and COL-X was assessed at 10 weeks post-injury [68]. Investigation by von der Mark and colleagues demonstrated an irregular distribution of COL-X that was localized around chondrocyte clusters in fibrillated OA cartilage and that was absent from the non-calcified cartilage region of normal articular cartilage [65]. COL-X localization and transient expression at sites of calcification suggest that it is associated with events in early stages of endochondral bone formation. COL-X gene expression was detected in chondrocytes present in $\mathrm{OA}$ tissue in that area where there appeared to be a re-initiation of the endochondral bone formation process including osteophytes and in the areas of subchondral bone sclerosis [69]. COL-X was present in hypertrophic chondrocytes in the vertebrae of P1 spine (Figure 11C).

Matrix metalloproteinase 13 (MMP13; Collagenase 3): Matrix metalloproteinase 13 (MMP13) or collagenase 3 is a member of the MMP family, and the MMPs belong to a larger family of proteases known as the metzincin superfamily. During embryonic development, MMP13 is expressed in the skeleton as required for restructuring the collagen matrix for bone mineralization. In pathological situations such as carcinomas, rheumatoid arthritis and $\mathrm{OA}$, it is highly overexpressed [70]. MMP13 is a major enzyme that targets cartilage for degradation. Compared to other MMPs, the expression of MMP13 is more restricted to connective tissue [71]. It not only targets COL-II in cartilage for degradation, but also degrades aggrecan, COL-IV and -IX, osteonectin and pelican in cartilage [72]. Patients with articular cartilage damage have high MMP13 expression suggesting that increased MMP13 is associated with cartilage degradation [73]. In the current study, MMP13 expression was higher in the MMx knee articular cartilage (Figures 12B-12B2) as compared to control knee articular cartilage (Figures 12A-12A2), indicating the success of our less invasive MMx procedure in causing damage to articular cartilage in rat.

Lubricin (PRG4): Lubricin (or proteoglycan 4, PRG4, superficial zone protein, SZP) is a mucinous glycoprotein and is secreted from synovial fibroblasts, and from chondrocytes in the superficial zone of articular 15 cartilage [74]. Lubricin lubricates the apposing and pressurized cartilage surfaces of the joint and provides chondroprotection [75]. In Prg4 knockout mice, surface cartilage of knee joint deteriorates, and the intimal cells become hyperplastic in the joint synovium [76]. Prg4 expressing cells, located at the joint surface at embryonic stage, serve as progenitors for all the deeper layers of mature articular cartilage. Prg4 expressing superficial chondrocytes, in young mice, expands into deeper regions of the articular cartilage as the animal age [77]. Alteration in joint mechanics or joint loading leads to altered lubricin expression in the superficial layer. Lubricin coating was reduced on the articular cartilage surface in meniscectomized sheep [78]. The intensive mechanical loading of knee joint, like running, induce Prg4 expression in the superficial zone of articular cartilage in mice [79]. The maximum Prg4-expressing articular cartilage progenitors occurred in the region of knee joints that experience the highest levels of mechanical loading, and thus lie in the central domains of the condyles [79]. In the current study, less invasive MMx may have caused more expression of lubricin in lateral articular cartilage of femur and tibia (Figure 13B1) and in medial articular cartilage of femur only (Figure 13B2). On the other hand, expression was minimum in the medial aspect of tibial cartilage, the region where the damage to articular cartilage was substantial due to fibrillation (Figure 13B2). The diffused PRG4 staining was found in the region from superficial tangential zone $(200 \mu \mathrm{m}$ deep) in OA articular cartilage in human patients [80]. The increased lubricin expression in $\mathrm{MMx}$ 
knee could be due the resistance of chondrocytes to additional tension due to MMx for the protection mechanism. In the fibrillated area, the tension had gone beyond limit, the addition of lubricin expression may not have helped, rather surface chondrocytes secreting lubricin, were sloughed off (Figure 13B2).

\section{Summary and Conclusion}

We applied less invasive approach of MMx to right knee of athymic rats and the left knee served as an unoperated control. At 10 weeks postMMx, MMx knee exhibited degenerative changes in articular cartilage and caused no significant subchondral bone damage. The less invasive $\mathrm{MMx}$ procedure, in rat, can be a model for early or less severe human OA due to articular cartilage degenerative changes only and with no subchondral bone degenerative changes in the knee synovial joint. The therapeutical drugs/agents designed to repair articular cartilage may be suitable for this model and may lead to treatment of early or less severe human OA.

\section{Acknowledgements}

The research was funded by Arthritis Program, University Health Network Toronto, Canada. The authors' thanks are due to Dr. Armand Keating and his lab members, Dr. Xing-Hua Wang, Amelie Chaboureau and Dr. Sowmya Viswanathan for providing me laboratory space and help; Miss Heather Whetstone (Ben Alman's Lab, Sickkids Hospital, Toronto), Dr. Keenan Thomas (Baylor College) Dr. Matthew Hilton and Dr. Bradley Estes (Duke University) for IHC questions Dr. Mohit Kapoor, Dr. Nizar Mohamed and Dr. Rajiv Gandhi for their continuous encouragement on research

\section{Conflict of Interest}

All the authors have nothing to declare.

\section{References}

1. Loeser RF (2010) Age-related changes in the musculoskeletal system and the development of osteoarthritis. Clin Geriatr Med 26: 371-386.

2. Eckstein F, Le Graverand MP (2015) Plain radiography or magnetic resonance imaging (MRI): Which is better in assessing outcome in clinical trials of diseasemodifying osteoarthritis drugs? Summary of a debate held at the World Congress of Osteoarthritis 2014. Semin Arthritis Rheum 45: 251-256.

3. Shen J, Li S, Chen D (2014) TGF-î² signaling and the development of osteoarthritis. Bone Res 2.

4. Pagnani MJ, Cooper DE, Warren RF (1991) Extrusion of the medial meniscus. Arthroscopy 7: 297-300.

5. McDermott I (2011) Meniscal tears, repairs and replacement: their relevance to osteoarthritis of the knee. Br J Sports Med 45: 292-297.

6. Zielinska B, Donahue TL (2006) 3D finite element model of meniscectomy: changes in joint contact behavior. J Biomech Eng 128: 115-123.

7. Bloecker K, Wirth W, Guermazi A, Hunter DJ, Resch H, et al. (2015) Medial meniscal extrusion relates to cartilage loss in specific femorotibial subregions: data from the osteoarthritis initiative. Arthritis Care Res (Hoboken) 67: 15451552.

8. Papalia R, Del Buono A, Osti L, Denaro V, Maffulli N (2011) Meniscectomy as a risk factor for knee osteoarthritis: a systematic review. Br Med Bull 99: 89-106.

9. Bendele AM (2001) Animal models of osteoarthritis. J Musculoskelet Neurona Interact 1: 363-376

10. Marshall KW, Chan AD (1996) Arthroscopic anterior cruciate ligament transection induces canine osteoarthritis. J Rheumatol 23: 338-343.

11. Janusz MJ, Bendele AM, Brown KK, Taiwo YO, Hsieh L, et al. (2002) Induction of osteoarthritis in the rat by surgical tear of the meniscus: Inhibition of joint damage by a matrix metalloproteinase inhibitor. Osteoarthritis Cartilage 10 : 785-791.

12. Hayami T, Pickarski M, Zhuo Y, Wesolowski GA, Rodan GA, et al. (2006) Characterization of articular cartilage and subchondral bone changes in the rat anterior cruciate ligament transection and meniscectomized models of osteoarthritis. Bone 38: 234-243.
13. Bomsta BD, Bridgewater LC, Seegmiller RE (2006) Premature osteoarthritis in the Disproportionate micromelia (Dmm) mouse. Osteoarthritis Cartilage 14 477-485.

14. Mason RM, Chambers MG, Flannelly J, Gaffen JD, Dudhia J, et al. (2001) The STR/ort mouse and its use as a model of osteoarthritis. Osteoarthritis Cartilage 9: 85-91.

15. Glasson SS, Blanchet TJ, Morris EA (2007) The surgical destabilization of the medial meniscus (DMM) model of osteoarthritis in the 129/SvEv mouse. Osteoarthritis Cartilage 15: 1061-1069.

16. Botter SM, van Osch GJ, Clockaerts S, Waarsing JH, Weinans H, et al. (2011) Osteoarthritis induction leads to early and temporal subchondral plate porosity in the tibial plateau of mice: an in vivo microfocal computed tomography study. Arthritis Rheum 63: 2690-2699.

17. Bendele AM, Hulman JF (1988) Spontaneous cartilage degeneration in guinea pigs. Arthritis Rheum 31: 561-565

18. Meacock SC, Bodmer JL, Billingham ME (1990) Experimental osteoarthritis in guinea-pigs. J Exp Pathol (Oxford) 71: 279-293.

19. Minns RJ, Muckle DS (1982) The role of the meniscus in an instability model for osteoarthritis in the rabbit knee. Br J Exp Pathol 63: 18-24.

20. Carlson CS, Loeser RF, Jayo MJ, Weaver DS, Adams MR, et al. (1994) Osteoarthritis in cynomolgus macaques: a primate model of naturally occurring disease. J Orthop Res 12: 331-339.

21. Piskin A, Gulbahar MY, Tomak Y, Gulman B, Hokelek M, et al. (2007) Osteoarthritis models after anterior cruciate ligament resection and medial meniscectomy in rats. A histological and immunohistochemical study. Saud Med J 28: 1796-1802.

22. Rezende MU, Hernandez AJ, Oliveira CR, Bolliger Neto R (2015) Experimenta osteoarthritis model by means of medial meniscectomy in rats and effects of diacerein administration and hyaluronic acid injection. Sao Paulo Med J 133 4-12.

23. Panahifar A, Maksymowych WP, Doschak MR (2012) Potential mechanism of alendronate inhibition of osteophyte formation in the rat model of post-traumatic osteoarthritis: evaluation of elemental strontium as a molecular tracer of bone formation. Osteoarthritis Cartilage 20: 694-702.

24. McErlain DD, Ulici V, Darling M, Gati JS, Pitelka V, et al. (2012) An in vivo investigation of the initiation and progression of subchondral cysts in a rodent model of secondary osteoarthritis. Arthritis Res Ther 14: R26

25. Intema F, Sniekers YH, Weinans H, Vianen ME, Yocum SA, et al. (2010) Similarities and discrepancies in subchondral bone structure in two differently induced canine models of osteoarthritis. J Bone Miner Res 25: 1650-1657.

26. Ene R, Sinescu RD, Ene P, Cîrstoiu MM, Cîrstoiu FC. (2015) Synovial inflammation in patients with different stages of knee osteoarthritis. Rom J Morphol Embryol 56: 169-173.

27. Van Lent PL, Van den Hoek AE, Van den Bersselaar LA, Spanjaards MF, Van Rooijen N, et al. (1993) In vivo role of phagocytic synovial lining cells in onset of experimental arthritis. Am J Pathol 143: 1226-1237.

28. Lai Y, Bai X, Zhao Y, Tian Q, Liu B, et al. (2014) ADAMTS-7 forms a positive feedback loop with TNF-Î \pm in the pathogenesis of osteoarthritis. Ann Rheum Dis 73: $1575-1584$

29. Christiansen BA, Guilak F, Lockwood KA, Olson SA, Pitsillides AA, et al. (2015) Non-invasive mouse models of post-traumatic osteoarthritis. Osteoarthritis Cartilage 23: 1627-1638.

30. Kamekura S, Hoshi K, Shimoaka T, Chung U, Chikuda H, et al. (2005) Osteoarthritis development in novel experimental mouse models induced by knee joint instability. Osteoarth Cartilage 13: 632-641.

31. Parfitt AM (1984) Age-related structural changes in trabecular and cortical bone: cellular mechanisms and biomechanical consequences. Calcif Tissue Int 36 Suppl 1: S123-128

32. McCalden RW, McGeough JA, Court-Brown CM (1997) Age-related changes in the compressive strength of cancellous bone. The relative importance of changes in density and trabecular architecture. J Bone Joint Surg Am 79: 421 427

33. Kadri A, Ea HK, Bazille C, Hannouche D, Liote F, et al. (2008) Osteoprotegerin inhibits cartilage degradation through an effect on trabecular bone in murine experimental osteoarthritis. Arthritis Rheum 58: 2379-2386. 
34. Juneja SC, Vonica A, Zeiss C, Lezon-Geyda K, Yatsula B, et al. (2014) Deletion of Mecom in mouse results in early-onset spinal deformity and osteopenia. Bone 60: 148-161.

35. Botter SM, van Osch GJ, Waarsing JH, van der Linden JC, Verhaar JA, et al. (2008) Cartilage damage pattern in relation to subchondral plate thickness in a collagenase-induced model of osteoarthritis. Osteoarthritis Cartilage 16: 506-514.

36. Lee H, Park Y, Ahn CW, Park SH, Jung EY, et al. (2014) Deer bone extract suppresses articular cartilage damage induced by monosodium iodoacetate in osteoarthritic rats: an in vivo micro-computed tomography study. J Med Food 17: 701-706.

37. Poole A (2005) Cartilage in health and disease. In: Koopman, W. and Moreland L. (eds), Arthritis \& Allied Conditions: A Textbook of Rheumatology (15th edn) Lippincott Williams, Wilkins, Philadelphia, PA, USA

38. Plaas A, Li J, Riesco J, Das R, Sandy JD, et al. (2011) Intraarticular injection of hyaluronan prevents cartilage erosion, periarticular fibrosis and mechanical allodynia and normalizes stance time in murine knee osteoarthritis. Arthritis Res Ther 13: R46.

39. Rosenberg $L$ (1971) Chemical basis for the histological use of safranin $O$ in the study of articular cartilage. J Bone Joint Surg Am 53: 69-82.

40. Pfander D, Heinz N, Rothe P, Carl HD, Swoboda B (2004) Tenascin and aggrecan expression by articular chondrocytes is influenced by interleukin 1beta: a possible explanation for the changes in matrix synthesis during osteoarthritis. Ann Rheum Dis 63: 240-244.

41. Li X, Kroin JS, Kc R, Gibson G, Chen D, et al. (2013) Altered spinal microRNA$146 \mathrm{a}$ and the microRNA-183 cluster contribute to osteoarthritic pain in knee joints. J Bone Miner Res 28: 2512-2522.

42. Junqueira LC, Bignolas G, Brentani RR (1979) Picrosirius staining plus polarization microscopy, a specific method for collagen detection in tissue sections. Histochem J 11: 447-455.

43. Montes GS, Junqueira LC (1991) The use of the Picrosirius-polarization method for the study of the biopathology of collagen. Mem Inst Oswaldo Cruz 86 Suppl 3: 1-11.

44. Dayan D, Hiss Y, Hirshberg A, Bubis JJ, Wolman M (1989) Are the polarization colors of picrosirius red-stained collagen determined only by the diameter of the fibers? Histochemistry 93: 27-29.

45. Aigner T, Stöve J (2003) Collagens--major component of the physiological cartilage matrix, major target of cartilage degeneration, major tool in cartilage repair. Adv Drug Deliv Rev 55: 1569-1593.

46. Bi X, Yang X, Bostrom MP, Bartusik D, Ramaswamy S, et al. (2007) Fourier transform infrared imaging and MR microscopy studies detect compositional and structural changes in cartilage in a rabbit model of osteoarthritis. Anal Bioanal Chem 387: 1601-1612.

47. Rieppo J, Hyttinen MM, Halmesmaki E, Ruotsalainen $H$, Vasara A, et al (2009) Changes in spatial collagen content and collagen network architecture in porcine articular cartilage during growth and maturation. Osteoarthritis Cartilage 17: 448-455.

48. Vasilceac FA, Renner AF, Teodoro WR, Mattiello-Rosa SM (2011) The remodeling of collagen fibers in rats ankles submitted to immobilization and muscle stretch protocol. Rheumatol Int 31: 737-742.

49. Nap RJ, Szleifer I (2008) Structure and interactions of aggrecans: statistical thermodynamic approach. Biophys J 95: 4570-4583.

50. Venn M, Maroudas A (1977) Chemical composition and swelling of normal and osteoarthrotic femoral head cartilage. I. Chemical composition. Ann Rheum Dis 36: 121-129.

51. Gleghorn L, Ramesar R, Beighton P, Wallis G (2005) A mutation in the variable repeat region of the aggrecan gene (AGC1) causes a form of spondyloepiphyseal dysplasia associated with severe, premature osteoarthritis. Am J Hum Genet 77: 484-490.

52. Henry SP, Liang S, Akdemir KC, de Crombrugghe B (2012) The postnatal role of Sox9 in cartilage. J Bone Miner Res 27: 2511-2525.

53. Aigner T, Vornehm SI, Zeiler G, Dudhia J, von der Mark K, et al. (1997) Suppression of cartilage matrix gene expression in upper zone chondrocytes of osteoarthritic cartilage. Arthritis Rheum 40: 562-569.

54. Kiani C, Chen L, Wu YJ, Yee AJ, Yang BB (2002) Structure and function of aggrecan. Cell Res 12: 19-32.
55. Lark MW, Gordy JT, Weidner JR, Ayala J, Kimura JH, et al. (1995) Cell-mediated catabolism of aggrecan. Evidence that cleavage at the "aggrecanase" site (Glu373-Ala374) is a primary event in proteolysis of the interglobular domain. J Biol Chem 270: 2550-2556.

56. Lark MW, Bayne EK, Flanagan J, Harper CF, Hoerrner LA, et al. (1997) Aggrecan degradation in human cartilage. Evidence for both matrix metalloproteinase and aggrecanase activity in normal, osteoarthritic, and rheumatoid joints. J Clin Invest 100: 93-106.

57. Janusz MJ, Little CB, King LE, Hookfin EB, Brown KK, et al. (2004) Detection of aggrecanase- and MMP-generated catabolic neoepitopes in the rat iodoacetate model of cartilage degeneration. Osteoarthritis Cartilage 12: 720-728.

58. Gibson GJ, Verner JJ, Nelson FR, Lin DL (2001) Degradation of the cartilage collagen matrix associated with changes in chondrocytes in osteoarthrosis. Assessment by loss of background fluorescence and immunodetection of matrix components. J Orthop Res 19: 33-42.

59. Carlson KM, Yamaga KM, Reinker KA, Hsia YE, Carpenter C, et al. (2006) Precocious osteoarthritis in a family with recurrent COL2A1 mutation. J Rheumatol 33: 1133-1136.

60. Kaufman GN, Zaouter C, Valteau B, Sirois P, Moldovan F (2011) Nociceptive tolerance is improved by bradykinin receptor B1 antagonism and join morphology is protected by both endothelin type A and bradykinin receptor B antagonism in a surgical model of osteoarthritis. Arthritis Res Ther 13: R76.

61. Xu Y, Dai GJ, Liu Q, Liu ZL, Song ZQ, et al. (2014) Sanmiao formula inhibits chondrocyte apoptosis and cartilage matrix degradation in a rat model of osteoarthritis. Exp Ther Med 8: 1065-1074.

62. Gross J, Nagai Y (1965) Specific degradation of the collagen molecule by tadpole collagenolytic enzyme. Proc Natl Acad Sci U S A 54: 1197-1204.

63. Welgus HG, Jeffrey JJ, Eisen AZ (1981) The collagen substrate specificity of human skin fibroblast collagenase. J Biol Chem 256: 9511-9515.

64. Grant WT, Wang GJ, Balian G (1987) Type X collagen synthesis during endochondral ossification in fracture repair. J Biol Chem 262: 9844-9849.

65. von der Mark K, Kirsch T, Nerlich A, Kuss A, Weseloh G, et al. (1992) Type X collagen synthesis in human osteoarthritic cartilage. Indication of chondrocyte hypertrophy. Arthritis Rheum 35: 806-811.

66. McIntosh I, Abbott MH, Francomano CA (1995) Concentration of mutation causing Schmid metaphyseal chondrodysplasia in the C-terminal noncollagenous domain of type $X$ collagen. Hum Mutat 5: 121-125.

67. Kwan KM, Pang MK, Zhou S, Cowan SK, Kong RY, et al. (1997) Abnorma compartmentalization of cartilage matrix components in mice lacking collagen X: implications for function. J Cell Biol 136: 459-471.

68. Stoop R, Buma P, van der Kraan PM, Hollander AP, Billinghurst RC, et al 2001. Type II collagen degradation in articular cartilage fibrillation after anterior cruciate ligament transection in rats. Osteoarthritis Cartilage 9: 308-315.

69. Hoyland JA, Thomas JT, Donn R, Marriott A, Ayad S, et al. (1991) Distribution of type $X$ collagen mRNA in normal and osteoarthritic human cartilage. Bone Miner 15: 151-163.

70. Sternlicht MD, Werb Z (2001) How matrix metalloproteinases regulate cell behavior. Annu Rev Cell Dev Biol 17: 463-516.

71. Vincenti MP, Brinckerhoff CE (2002) Transcriptional regulation of collagenase (MMP-1, MMP-13) genes in arthritis: integration of complex signaling pathways for the recruitment of gene-specific transcription factors. Arthritis Res 4: 157-

72. Shiomi T, Lemaître V, D'Armiento J, Okada Y (2010) Matrix metalloproteinases, a disintegrin and metalloproteinases, and a disintegrin and metalloproteinases with thrombospondin motifs in non-neoplastic diseases. Pathol Int 60: 477-496.

73. Roach HI, Yamada N, Cheung KS, Tilley S, Clarke NM, et al. (2005) Association between the abnormal expression of matrix-degrading enzymes by human osteoarthritic chondrocytes and demethylation of specific $\mathrm{CpG}$ sites in the promoter regions. Arthritis Rheum 52: 3110-3124.

74. Jay GD, Britt DE, Cha CJ (2000) Lubricin is a product of megakaryocyte stimulating factor gene expression by human synovial fibroblasts. J Rheumato 27: $594-600$

75. Jay GD (1992) Characterization of a bovine synovial fluid lubricating factor. I. Chemical, surface activity and lubricating properties. Connect Tissue Res 28 : 71-88 
Citation: Juneja SC, Ventura M, Jay GD, Veillette C (2016) A Less Invasive Approach of Medial Meniscectomy in Rat: A Model to Target Early or Less Severe Human Osteoarthritis. J Arthritis 5: 193. doi:10.4172/2167-7921.1000193

76. Rhee DK, Marcelino J, Baker M, Gong Y, Smits P, et al. (2005) The secreted glycoprotein lubricin protects cartilage surfaces and inhibits synovial cell overgrowth. J Clin Invest 115: 622-631.

77. Kozhemyakina E, Zhang M, lonescu A, Ayturk UM, Ono N, et al. (2015) Identification of a Prg4-expressing articular cartilage progenitor cell population in mice. Arthritis Rheumatol 67: 1261-1273.

78. Young AA, McLennan S, Smith MM, Smith SM, Cake MA, et al. (2006) Proteoglycan 4 downregulation in a sheep meniscectomy model of early osteoarthritis. Arthritis Res Ther 8: R41.
79. Ogawa H, Kozhemyakina E, Hung HH, Grodzinsky AJ, Lassar AB (2014) Mechanical motion promotes expression of Prg4 in articular cartilage via multiple CREB-dependent, fluid flow shear stress-induced signaling pathways. Genes Dev 28: 127-139.

80. Zhang D, Johnson LJ, Hsu HP, Spector M (2007) Cartilaginous deposits in subchondral bone in regions of exposed bone in osteoarthritis of the human knee: histomorphometric study of PRG4 distribution in osteoarthritic cartilage. J Orthop Res 25: 873-883. 